\title{
FANO 4-FOLDS WITH SCROLL STRUCTURE
}

\author{
ADRIAN LANGER
}

\begin{abstract}
We classify smooth Fano 4-folds with second betti number $b_{2}=2$, possesing adjunction theoretic scroll structure. These manifolds occur to be projectivisations of coherent sheaves over Fano manifolds. The paper deals mainly with projectivisations of non-locally free rank 2 Fano sheaves over Fano 3 -folds with $b_{2}=1$, using Mori theory. By the way, we classify nef and big rank 2 bundles over $\mathbf{P}^{2}$ with the first Chern class $\mathcal{O}(3)$.
\end{abstract}

\section{Introduction}

In this paper we classsify smooth Fano 4 -folds with second Betti number $b_{2}=2$ which possess scroll structure (in the sense of adjunction theory). It turns out that such manifolds must be projectivisations of coherent sheaves over some smooth Fano varieties of dimension less or equal to 3. The case when dimension of the base is equal to 1 is very easy. The case of dimension 2 was studied in [SzW3] (let us note that $\mathbf{P}^{2}$ is the only Fano surface with $b_{2}=1$ ). Here we classify such structures over base of dimension 3 , which completes above mentioned classification. In view of the latest progress in classification of contractions of 4-folds (see [AW2]), this can be treated as the first step towards a classification of Fano 4-folds in general.

Our approach to the problem of classification is slightly different from the one used until now in papers [SzW1], [SzW2], [SzW3]. We get our results by computing possible Chern classes by means of numerical properties and then by vanishing theorems and "sheaf" methods (using known facts about stable sheaves and jumping lines). Such an approach also allows to obtain known results about Fano bundles with little knowledge of stable bundles. As an example, in appendix we compute Fano bundles of rank 2 on $\mathbf{P}^{2}$ without using Iskovskich-Mori-Mukai list of all Fano threefolds.

The first step towards the classification, i.e. computation of Chern classes of sheaves $\mathcal{E}$, is divided into three parts according to the type of second contraction $\phi$ of $\mathbf{P}(\mathcal{E})$. If this morphism $\phi$ is onto a variety $Y$ of dimension less than 4 , then computation is very easy. We simply know that

Received September 12, 1995. 
the self intersection of a pull back of an ample divisor from $Y$ is zero, which is nearly sufficient to compute possible Chern classes of $\mathcal{E}$. The case of a divisorial contraction is more complicated and we have to use also some well known results of Ando [A] in order to determine good supporting divisor of $\phi$. The third part concerns small contractions, whose structure is known by results of Kawamata [Ka]. Nevertheless the computation in this last case is not obvious and easy (at least to the author). Next steps in classification are geometrical considerations, concerning sheaves and bundles, depending mainly on cohomology theory. We also discuss geometry of projectivisation of obtained sheaves.

We have to deal separately with Fano sheaves on different Fano 3-folds, according to the index of this 3 -fold. The paper is divided into 9 sections. The first two sections deal with some useful general facts and definitions. In section 3 we classify nef and big rank 2 vector bundles over $\mathbf{P}^{2}$. This is used in sections 4-6 for the classification of rank 2 Fano sheaves over $\mathbf{P}^{3}$. The results concerning sheaves on other Fano 3-folds are briefly treated in sections 7 and 8 . Finally in section 9 we describe the geometry of obtained Fano 4-folds.

Acknowledgements. I would like to thank to Prof. J. Wiśniewski for very valuable discussions, advices and for help while preparing this paper. I would also like to acknowledge partial support from Polish KBN.

\section{$\S 1 . \quad$ Preliminaries}

In this section we recall some definitions and results which will be used later on. Notation and terminology used in this paper is consistent with the one used in [Ha1] and previous papers concerning this topic (cf. [SzW1], [SzW2] and [BW]). The only difference is using the notation $\left.\mathcal{E}\right|_{H}$ for an $\mathcal{O}_{H^{-}}$ module associated to a restriction of $\mathcal{E}$ to subvariety $H$.

Locally free sheaves and vector bundles are not distinguished, similarly as divisors and line bundles on smooth manifolds. All varieties are assumed to be defined over complex number field.

\section{1 .}

Let $X$ be a 3-dimensional smooth Fano variety with the second Betti number $b_{2}(X)=1$. Let us denote by $H$ a class of the generator of Picard group Pic $X$. From the assumptions one can write $-K_{X}=r(X) \cdot H$ for some integer $r(X)$ called the index of $X$. It is known by the result of Shokurov that on every such manifold there exists at least 1-dimensional 
family of lines. By a line we mean here a smooth rational curve $L$ such that $L \cdot H=1$. Hence $H^{2}(X, \mathbf{Z})$ is generated by the class $H, H^{4}(X, \mathbf{Z})$ is generated by a line $L$ and $H^{6}(X, \mathbf{Z})$ by a point $P$. Therefore one may identify in the known way, Chern classes of sheaves on $X$ with integers.

Let $\mathcal{F}$ be a coherent sheaf of rank $r$ on $X$. From the Riemann-Roch formula it follows that:

$$
\begin{aligned}
\chi(\mathcal{F})=\frac{1}{6}\left(c_{1}{ }^{3} H^{3}-3 c_{1} c_{2}+3 c_{3}\right)+\frac{1}{4} r(X) & \left(c_{1}{ }^{2} H^{3}-2 c_{2}\right) \\
+ & \frac{1}{12} c_{1}\left(r(X)^{2} H^{3}+\frac{24}{r(X)}\right)+r
\end{aligned}
$$

where $c_{1}, c_{2}, c_{3}$ stand for Chern classes of $\mathcal{F}$. In what follows, we will extensively use this formula for $\mathbf{P}^{3}$, so for the convenience of the reader, we will write this formula in explicit form for sheaf $\mathcal{F}$ on $\mathbf{P}^{3}$ :

$$
\chi(\mathcal{F})=r-1+\left(\begin{array}{c}
c_{1}+3 \\
3
\end{array}\right)-2 c_{2}+\frac{1}{2}\left(c_{3}-c_{1} c_{2}\right)
$$

For $\mathcal{F}$ of rank $r=2$ on $\mathbf{P}^{3}$ we have $c_{1} c_{2} \equiv c_{3}(\bmod 2)$ and

$$
\begin{aligned}
& c_{1}(\mathcal{F}(k))=c_{1}+2 k \\
& c_{2}(\mathcal{F}(k))=c_{2}+c_{1} k+k^{2} \\
& c_{3}(\mathcal{F}(k))=c_{3} .
\end{aligned}
$$

Another simple fact which we use is the existence of an isomorphism:

$$
(\mathcal{F})^{*} \simeq \mathcal{F}\left(-c_{1}\right)
$$

for a rank 2 reflexive sheaf $\mathcal{F}$.

\section{2.}

In this subsection we recall some results and definitions from [BW].

DEFINITION 1.2.1. A coherent sheaf $\mathcal{E}$ of rank $r \geq 2$ over a normal variety $Y$ is called Bănică sheaf if its projectivization is a smooth variety.

Proposition 1.2.2. If $\mathcal{E}$ is a Bănică sheaf then it is reflexive, the projection $p: \mathbf{P}(\mathcal{E}) \rightarrow Y$ is an extremal ray contraction and $\mathcal{O}_{\mathbf{P}(\mathcal{E})}(1)$ is $p$ ample. 
DEFINITION 1.2.3. We say that a coherent sheaf $\mathcal{E}$ over a normal variety $Y$ extends to a locally free sheaf $\mathcal{F}$ if there exists a sequence of $\mathcal{O}_{Y}$-modules

$$
0 \longrightarrow \mathcal{O} \longrightarrow \mathcal{F} \longrightarrow \mathcal{E} \longrightarrow 0
$$

Proposition 1.2.4. Let $\mathcal{E}$ be a Bănică sheaf of rank $n-1$ over $a$ smooth projective variety $Y$ of dimension n. If $H^{2}\left(Y, \mathcal{E}^{*}\right)=0$, then $\mathcal{E}$ extends to a locally free sheaf; in particular the sheaf $\mathcal{E} \otimes \mathcal{L}^{-m}$ extends for an ample line bundle $\mathcal{L}$ and $m \gg 0$.

THEOREM 1.2.5. Let $\mathcal{E}$ be a Bănică sheaf of rank $r$ on a normal variety $Y$. If $r \geq \operatorname{dim} Y$, then $Y$ is smooth and $\mathcal{E}$ is locally free.

\section{3.}

In this paper we use the following version of the relative KawamataViehweg theorem (see [KMM, theorem 1.2.5]).

THEOREM 1.3.1. Let $X$ be a smooth manifold and $\pi: X \rightarrow Y$ be a proper morphism onto a variety $Y$. Assume that $D-K_{X}$ is $\pi$-nef and big for some divisor $D$ on $X$. Then $R^{i} \pi_{*} \mathcal{O}_{X}(D)=0$ for $i>0$.

In section 3 we use the following theorem (see $[\mathrm{Re}]$ ):

THEOREM 1.3.2. Let $X$ be a projective variety with only canonical singularities, $D \in \operatorname{Pic} X-a$ nef divisor and $a D-K_{X}-a$ big and nef divisor for some $a \geq 1$. Then for $m \gg 0|m D|$ is base point free and defines $a$ morphism $\phi: X \rightarrow Y$ on a normal variety $Y$ such that $\phi_{*} \mathcal{O}_{X}=\mathcal{O}_{Y}$ and there exists an ample divisor $A$ on $Y$ such that $D=\phi^{*} A$. Moreover if $D$ is big then $\phi$ is birational and $Y$ has only rational singularities.

\section{4.}

Let us recall a couple of theorems from Mori theory - a detailed survey of this theory can be found in [KMM].

Theorem 1.4.1. (Kawamata-Shokurov) For any extremal ray $R$ on a $\mathbf{Q}$-factorial projective variety $X$ with canonical singularities there exists a normal projective variety $Y$ and a surjective morphism $\phi: X \rightarrow Y$ with connected fibers that contracts a curve $C$ if and only if $[C] \in R$. Such a morphism we call an extremal ray contraction. 
Proposition 1.4.2. If $X$ is a Fano manifold, then the cone of effective curves $N E(X)$ is a rational polyhedral cone spanned by a finite number of extremal rays.

1.4.3. One can distinguish three types of a ray contraction $\phi: X \rightarrow$ $Y$ according to the dimension of the contracted set $E(\phi):=\{x \in X$ : $\left.\operatorname{dim} \phi^{-1}(\phi(x))>0\right\}$ :

- a fiber contraction, if $E(\phi)=X$. Then $X$ is covered by rational curves and a general fiber of $\phi$ is a Fano variety.

- a divisorial contraction, if $\operatorname{codim} E(\phi)=1$. Then $Y$ is a $\mathbf{Q}$-factorial projective variety with canonical singularities.

- a small contraction, if $\operatorname{codim} E(\phi)>1$. In this case $Y$ has to have non $\mathbf{Q}$-factorial singularities.

\section{5.}

In the present paper we will study Fano sheaves on smooth 3-dimensional Fano varieties. One motivation for studying such sheaves comes from the following. Let $X$ be a smooth 4-dimensional Fano variety admitting a scroll structure $p: X \rightarrow Z$, without divisorial fibers, over a normal variety $Z$. Then from [AW, theorem 4.1 and remark 4.12] it follows that $Z$ is smooth and $X$ is a projectivisation of a coherent sheaf from $Z$. Moreover, from $[\mathrm{BW}$, lemma 3.4] it follows that $Z$ is a Fano variety. Now we know that $p$ is an extremal ray contraction, and if we assume $b_{2}(X)=2$, then $b_{2}(Z)=1$. If the dimension of $Z$ is at most 2 , then $X$ is a projective bundle of rank ( $\operatorname{dim} X-\operatorname{dim} Z+1)$ over $Z$, and $Z$ is the projective space $\mathbf{P}^{1}$ or $\mathbf{P}^{2}$. In the first case one can easily check that $X=\mathbf{P}_{\mathbf{P}^{1}}\left(\mathcal{O}^{4}\right)$ or $X=\mathbf{P}_{\mathbf{P}^{1}}\left(\mathcal{O}^{3} \oplus \mathcal{O}(1)\right)$. The second case was studied by Szurek and Wiśniewski in [SzW3] and completely settled. In this paper we study the case: $\operatorname{dim} Z=3$. We will completely classify all such manifolds $X$, some of which occur to be projectivisations of non-locally free sheaves.

We will divide studying of Fano sheaves on Fano 3-folds according to the index $r(X)$ of $X$. The main idea in all cases remains the same, but there are some differences in numerical data. We use here classification of Fano threefolds in essential way (in particular we use the bound for the degree of Fano 3-folds of index 1). Fano threefolds of index 2 and degree $d$ are denoted by $V_{d}$. The next section is devoted to some general facts and then in sections 3-6 we deal with technically the most difficult case of sheaves on $\mathbf{P}^{3}$. 


\section{§2. Ample sheaves and vanishing theorems}

Let us recall that a sheaf $\mathcal{E}$ is ample (resp. nef, big) if $\mathcal{O}(1)$ is ample (resp. nef, big) on $\mathbf{P}(\mathcal{E})$.

LEMMA 2.1. Let $\mathcal{E}$ be a Bănică sheaf over a smooth projective variety $Y$ and let $p: \mathbf{P}(\mathcal{E}) \rightarrow Y$ be a natural projection. Then for any coherent sheaf $\mathcal{F}$ on $Y$ and any $n \gg 0$ there is a natural isomorphism

$$
\mathcal{F} \otimes\left(S^{n}(\mathcal{E})\right)^{* *} \longrightarrow p_{*}\left(p^{*}(\mathcal{F}) \otimes \mathcal{O}_{\mathbf{P}(\mathcal{E})}(n)\right)
$$

and the following equality

$$
R^{i} p_{*}\left(p^{*}(\mathcal{F}) \otimes \mathcal{O}_{\mathbf{P}(\mathcal{E})}(n)\right)=0
$$

holds for $i>0$.

Proof. The same as in the case of vector bundles (cf. [Ha2, lemma 3.1]). It should be only proved that $R^{i} p_{*}\left(\mathcal{O}_{X}(n)\right)=0$ for $i>0, n \geq 0$. This equality follows immediately from the theorem 1.3.1.

Corollary 2.2. Let $\mathcal{E}$ be a Bănică sheaf of rank 2 on $\mathbf{P}^{3}$. If $c_{1}(\mathcal{E})=$ 0,1 and $\mathcal{E}(1)$ is nef and big then $H^{i}\left(\mathbf{P}^{3}, \mathcal{E}\right)=0$ for $i>0$.

Proof. It follows from the lemma, Leray spectral sequence and Kawamata-Viehweg vanishing theorem on $\mathbf{P}(\mathcal{E})$.

Remark. The vanishing theorem of le Potier (see [SS, theorem 5.17]) fails for non-locally free sheaves even if the sheaf is Bănică with isolated singularities, i.e., it is not generally true that if $K^{*} \otimes \mathcal{E}$ is ample then $H^{q}(X, \mathcal{E})=0$ for $q \geq r=\operatorname{rank} \mathcal{E}$. A simple counterexample to this expectation gives the following sheaf $\mathcal{E}$ of rank 2 on $\mathbf{P}^{3}$ :

$$
0 \longrightarrow \mathcal{O}(-1) \longrightarrow \mathcal{O}^{3} \longrightarrow \mathcal{E}(3) \longrightarrow 0
$$

as $K^{*} \otimes \mathcal{E}$ is ample but $h^{2}\left(\mathbf{P}^{3}, \mathcal{E}\right)=1 \neq 0$.

THEOREM 2.3. Let $\mathcal{E}$ be as in lemma 2.1. The following statements are equivalent:

1) the sheaf $\mathcal{E}$ is ample, 
2) for any coherent sheaf $\mathcal{F}$ the sheaf $\mathcal{F} \otimes\left(S^{n}(\mathcal{E})\right)^{* *}$ is globally generated by global sections for $n \gg 0$.

Proof. The same as the proof of [Ha2, theorem 3.2] (the assumption on projectivity of $Y$ is valid).

Lemma 2.4. Assume that $\mathcal{F}$ is a coherent sheaf on $\mathbf{P}^{n}$ with zerodimensional singular locus, $n \geq 3$ and the homological dimension of the sheaf $\mathcal{F}$ is at most 1 . Moreover let us assume that $\left.\mathcal{F}\right|_{\mathbf{p}^{2}}$ is a decomposable bundle for some $\mathbf{P}^{2}$. Then the sheaf $\mathcal{F}$ is also a decomposable bundle.

Proof. (cf. [OSS, I, theorem 2.3.2] and [SRS, theorem 2.5]) For general $\mathbf{P}^{n-1}$ containing $\mathbf{P}^{2}$ from the assumption $\left.\mathcal{F}\right|_{\mathbf{P}^{n-1}}$ is locally free and splits (this needs some easy extra argument). In particular from Horrock's criterion: $H^{\imath}\left(\mathbf{P}^{n-1},\left.\mathcal{F}\right|_{\mathbf{P}^{n-1}}(k)\right)=0$ for $0<i<n-1, k \in \mathbf{Z}$.

Let us consider the divisorial sequence:

$$
0 \longrightarrow \mathcal{F}(k-1) \longrightarrow \mathcal{F}(k) \longrightarrow\left(\left.\mathcal{F}\right|_{\mathbf{P}^{n-1}}\right)(k) \longrightarrow 0 .
$$

Similarly as in [OSS] $H^{i}\left(\mathbf{P}^{n}, \mathcal{F}(k)\right)=0$ and $H^{i}\left(\mathbf{P}^{n}, \mathcal{F}^{*}(k)\right)=0$ for $2 \leq i \leq$ $n-1$ and $k \in \mathbf{Z}$. Now let us look at the spectral sequence of local and global Ext functors:

$$
E_{2}^{p q}=H^{p}\left(\operatorname{Ext}^{q}(\mathcal{F}, \mathcal{O})\right) \Rightarrow E^{p+q}=\operatorname{Ext}^{p+q}(\mathcal{F}, \mathcal{O}) .
$$

From our assumptions $\operatorname{Ext}^{q}(\mathcal{F}, \mathcal{O})=0$ for $q \geq 2$ and $\operatorname{Ext}^{1}(\mathcal{F}, \mathcal{O})$ is a coherent sheaf with support at points where $\mathcal{F}$ is not locally free.

It follows that $E_{2}^{p q}=0$ for $q \geq 2$ and $q=1, p \geq 1$. Now from the Serre duality theorem there is the following exact sequence:

$$
\begin{aligned}
0 \rightarrow H^{1}\left(\mathcal{F}^{*}\right) \longrightarrow H^{n-1}(\mathcal{F} \otimes \omega)^{\prime} & \longrightarrow H^{0}\left(\operatorname{Ext}^{1}(\mathcal{F}, \mathcal{O})\right) \\
& \longrightarrow H^{2}\left(\mathcal{F}^{*}\right) \longrightarrow H^{n-2}(\mathcal{F} \otimes \omega)^{\prime} \rightarrow 0 .
\end{aligned}
$$

Therefore $\operatorname{Ext}^{1}(\mathcal{F}, \mathcal{O})=0$ and $\mathcal{F}$ is locally free. Hence $\mathcal{F}$ splits.

We will need the following easy, technical lemma in the sequel:

Lemma 2.4.1. Let $\mathcal{E}$ be a Bănică sheaf of rank 2 on $\mathbf{P}^{3}$. Let us assume that $h^{1}(\mathcal{E})=h^{2}(\mathcal{E})=0$ and for some $\mathbf{P}^{2}$ which does not contain singular points of $\mathcal{E}$ we have $h^{1}\left(\left.\mathcal{E}\right|_{\mathbf{p}^{2}}\right)=1$. Let $\mathcal{F}$ be a non-zero element of $\operatorname{Ext}^{1}\left(\left.\mathcal{E}(k)\right|_{\mathbf{P}^{2}}, \mathcal{O}_{\mathbf{P}^{2}}(k-3)\right)$, and $\mathcal{G}$ be a non-zero element of $\operatorname{Ext}^{1}(\mathcal{E}(k), \mathcal{O}(k-$ 3)) for some integer $k$. Then it follows that $\left.\mathcal{G}\right|_{\mathbf{P}^{2}}=\mathcal{F}$. If moreover $\mathcal{F}$ splits on $\mathbf{P}^{2}$, then $\mathcal{G}$ splits too. 
Proof. The divisorial sequence:

$$
\left.0 \longrightarrow \mathcal{E}(-1) \longrightarrow \mathcal{E} \longrightarrow \mathcal{E}\right|_{\mathbf{P}^{2}} \longrightarrow 0 .
$$

gives an exact cohomology sequence:

$$
0=H^{1}(\mathcal{E}) \longrightarrow H^{1}\left(\left.\mathcal{E}\right|_{\mathbf{P}^{2}}\right) \stackrel{\delta}{\longrightarrow} H^{2}(\mathcal{E}(-1)) \longrightarrow H^{2}(\mathcal{E})=0 .
$$

Now let us consider the following diagram:

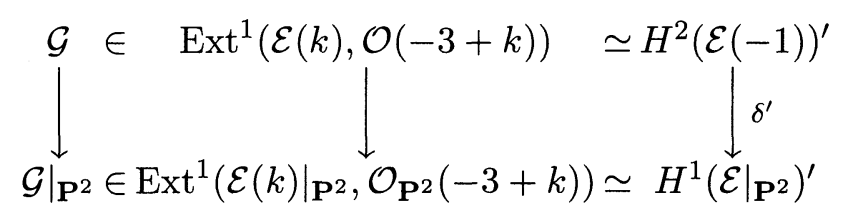

Since $\delta$ is an isomorphism, $\delta^{\prime}$ is also an isomorphism, $\operatorname{dim} \operatorname{Ext}^{1}(\mathcal{E}(k), \mathcal{O}(-3+$ $k))=1$ and $\left.\mathcal{G}\right|_{\mathbf{P}^{2}}=\mathcal{F}$. The last statement of the lemma is obvious because of lemma 2.4 .

Suppose that $\mathcal{E}$ is a vector bundle over a smooth manifold $X$. Then there is a Leray-Hirsch formula allowing us to compute the intersection product on the projectivisation $\mathbf{P}(\mathcal{E})$. In general there is no such formula for sheaves but in the case of sheaves of $\operatorname{rank} \operatorname{dim} X-1$ we have the following lemma which allows us to compute intersections on $\mathbf{P}(\mathcal{E})$. Below $B^{*}(X)$ denotes the ring of algebraic equivalence classes of cycles on $X$ and $B^{k}(X)$ stands for a group of classes of codimension $k$ cycles.

Lemma 2.5. Let $\mathcal{E}$ be a rank $r=n-1$ Bănică sheaf on a smooth manifold $X$ of dimension $n$ and let $F_{1}, \ldots, F_{k}$ be the fibers of $p: \mathbf{P}(\mathcal{E}) \longrightarrow X$, corresponding to singular points of the sheaf $\mathcal{E}$. Denote $k=c_{n}(\mathcal{E})$. Then

$$
B^{*}(\mathbf{P}(\mathcal{E}))=\left(B^{*}(X)[\xi] \oplus \bigoplus_{i=1}^{k} F_{i}\right) / R
$$

Here $R$ denotes the following relations: $\sum_{i=0}^{r}(-1)^{i} p^{*} c_{i}(\mathcal{E}) \xi^{r-i}=(-1)^{r} \sum_{i=1}^{k}$ $F_{i}, F_{i} F_{j}=0$ for $i \neq j, F_{i}^{2}=(-1)^{r}, F_{i} \xi=p^{*}(x)$, where $x$ is a general point of $X$, and $F_{i} \cdot p^{*}(V)=0$ for any $V \in B^{*}(Y)$.

Proof. Let $p_{1}, \ldots, p_{k}$ denote singular points of $\mathcal{E}$ and let $Z=\bigcup_{i=1}^{k} F_{k}$, $T=Y-\left\{p_{1}, \ldots, p_{k}\right\}, U=X-Z$. Of course $F_{i} F_{j}=0$ for $i \neq j$ and 
$F_{i} \xi=p^{*}(x)$, because $\left.\xi\right|_{F_{i}}=\mathcal{O}_{\mathbf{P}^{r}}(1)$. Denote $i: Z \hookrightarrow X, j: U \longrightarrow X$. From [Ful, example 10.3.4] there is the following exact sequence:

$$
B(Z) \longrightarrow B(X) \longrightarrow B(U) \longrightarrow 0
$$

It is known that:

$$
B(U)=B(T)[\xi] /\left(\sum_{i=0}^{r}(-1)^{i} p^{*} c_{i}(\mathcal{E}) \cdot \xi^{r-i}=0\right) .
$$

Therefore in $B(X)$ :

$$
\sum_{i=0}^{r}(-1)^{i} p^{*} c_{i}(\mathcal{E}) \cdot \xi^{r-i}=\sum_{i=1}^{k} a_{i} F_{i} \in \operatorname{ker} j^{*}=\operatorname{im} i_{*}
$$

for some $a_{i}$. Intersecting both sides of this equation with $F_{i}$ we obtain:

$$
a_{i} F_{i}^{2}=\xi^{r} F_{i}=1 \text {. }
$$

Now let $\mathcal{F}$ denote an extension of $\mathcal{E}$ by $\mathcal{O}(k)$ for some $k \gg 0$. We have the following exact sequence:

$$
\left.0 \rightarrow N_{F_{2} / \mathbf{P}(\mathcal{E})} \longrightarrow N_{F_{i} / \mathbf{P}(\mathcal{F})} \longrightarrow N_{\mathbf{P}(\mathcal{E}) / \mathbf{P}(\mathcal{F})}\right|_{F_{i}} \rightarrow 0
$$

Here $\mathbf{P}(\mathcal{E})$ is a smooth divisor from the linear system $\left|\mathcal{O}_{\mathbf{P}(\mathcal{F})}\left(\xi_{\mathcal{F}}-k H\right)\right|$, $\left.\mathcal{O}\left(\xi_{\mathcal{F}}\right)\right|_{F_{i}}=\mathcal{O}(1)$ and $\left.\mathcal{O}(H)\right|_{F_{i}}=\mathcal{O}$, so $\left.N_{\mathbf{P}(\mathcal{E}) / \mathbf{P}(\mathcal{F})}\right|_{F_{2}}=\mathcal{O}_{F_{i}}(1)$. We also know that $N_{F_{i} / \mathbf{P}(\mathcal{F})}=\mathcal{O}_{F_{i}}^{r+1}$, hence $N_{F_{2} / X} \simeq \Omega_{F_{i}}(1)$ and $F_{i}^{2}=c_{r}\left(N_{F_{i} / X}\right)=$ $(-1)^{r}=a_{i}$.

Since $F_{i} \cap \bar{U}=h \in \operatorname{Pic} F_{i}$ generates $\operatorname{Pic} F_{i}$ and it is algebraically equivalent to subvarieties of $U$ not intersecting $h$ we see that $h=0$ in $B^{*}(X)$. Now $B(X)$ is generated by $\xi, F_{1}, \ldots, F_{k}$ and $B(Y)$ as $\mathbf{Z}$-algebra, so it is straightforward that there are no other relations in $B(X)$.

Corollary 2.6. Let $\mathcal{E}$ be a Bănică sheaf of rank 2 on a Fano 3-fold with $b_{2}=1$. Let $h$ be a generator of the Picard group and $h^{3}=d$. Then:

$$
H^{4}=0, H^{3} \xi=d, H^{2} \xi^{2}=d c_{1}, H \xi^{3}=d c_{1}^{2}-c_{2}, \xi^{4}=d c_{1}^{3}-2 c_{1} c_{2}+c_{3},
$$

where $H$ denotes a pull back of $h$.

Proof. It is immediately obtained from the previous theorem and an obvious remark that $H^{4}=0$ and $H^{3} \xi=d$. 
Lemma 2.7. Let $\mathcal{E}$ be a rank 2 Fano sheaf on $\mathbf{P}^{3}$ and $a H+b \xi$ be $a$ nef divisor on $\mathbf{P}(\mathcal{E})$. If $c_{1}=0$ or 1 , then $a \geq 0, b \geq 0$.

Proof. It is easy to see that $b \geq 0$. So let us assume that $a<0$. Then $(a H+b \xi)+(-a) H$ is ample, hence $\mathcal{E}$ is an ample sheaf. If we restrict $\mathcal{E}$ to the general line $L$ we obtain:

$$
\left.\mathcal{E}\right|_{L}=\mathcal{O}_{L}(c) \oplus \mathcal{O}_{L}(d), \quad c+d=c_{1}, \text { where } c, d>0
$$

which is impossible.

$\S$ 3. Classification of rank 2 weak Fano sheaves on $\mathbf{P}^{2}$ with $c_{1}=-1$

Definition 3.1. A sheaf $\mathcal{E}$ on $X$ is weak Fano if $\mathbf{P}(\mathcal{E})$ is a smooth weak Fano variety, i.e., $-K_{\mathbf{P}(\mathcal{E})}$ is nef and big.

This is a natural generalization of Fano sheaves. We will use it in classification of rank 2 Fano sheaves on $\mathbf{P}^{3}$. It occurs that the restriction of such sheaf to a general plane is weak Fano.

In this section the following notation is used: $\mathcal{E}$ is a weak Fano sheaf of rank 2 on $\mathbf{P}^{2}, c_{1}(\mathcal{E})=-1, X=\mathbf{P}(\mathcal{E}), p: X \longrightarrow \mathbf{P}^{2}$ a natural projection, $H=p^{*}\left(\mathcal{O}_{\mathbf{P}^{2}}(1)\right), \xi$ the divisor $\mathcal{O}_{\mathbf{P}(\mathcal{E})}(1)$ on $X, \phi: X \longrightarrow Y$ a "contraction" from theorem 1.3.2 for $D=\xi+2 H\left(-K_{X}=2 D\right.$ is nef and big, so the assumptions of the theorem are valid), $A$ an ample divisor on $Y$, such that $\phi^{*} A=D$.

THEOREM 3.2. Let $\mathcal{E}$ be a rank 2 weak Fano sheaf on $\mathbf{P}^{2}, c_{1}(\mathcal{E})=-1$. Then $\mathcal{E}(2)$ is globally generated and $\mathcal{E}$ is of the form:

$c_{2}(\mathcal{E})$

1. $-2, \quad \mathcal{O}(1) \oplus \mathcal{O}(-2)$

2. $0, \quad \mathcal{O} \oplus \mathcal{O}(-1)$

3. $1, \quad 0 \rightarrow \mathcal{O} \rightarrow \mathcal{E} \rightarrow J_{x}(-1) \rightarrow 0, x \in P^{2}$

or equivalently:

$0 \rightarrow \mathcal{O}(-1) \rightarrow \mathcal{O}^{2} \oplus \mathcal{O}(2) \rightarrow \mathcal{E}(2) \rightarrow 0$

4. $1, T_{P^{2}}(-2)$

5. $\quad 2, \quad 0 \rightarrow \mathcal{O}(-1) \rightarrow \mathcal{O}(1)^{2} \oplus \mathcal{O} \rightarrow \mathcal{E}(2) \rightarrow 0$ 
6. $3, \quad 0 \rightarrow \mathcal{O}(-1)^{2} \rightarrow \mathcal{O}(1) \oplus \mathcal{O}^{3} \rightarrow \mathcal{E}(2) \rightarrow 0$

7. $4, \quad 0 \rightarrow \mathcal{O}(-1)^{3} \rightarrow \mathcal{O}^{5} \rightarrow \mathcal{E}(2) \rightarrow 0$

8. $\quad 4, \quad 0 \rightarrow \mathcal{O}(-2) \rightarrow \mathcal{O}(1) \oplus \mathcal{O}^{2} \rightarrow \mathcal{E}(2) \rightarrow 0$

9. $5, \quad 0 \rightarrow \mathcal{O}(-1) \oplus \mathcal{O}(-2) \rightarrow \mathcal{O}^{4} \rightarrow \mathcal{E}(2) \rightarrow 0$

Proof.

3.2.0. Let us recall the intersections of divisors on $X$ :

$$
H^{3}=0, H^{2} \xi=1, H \xi^{2}=-1, \xi^{3}=1-c_{2}
$$

Since $0<\left(-K_{X}\right)^{3}=8\left(7-c_{2}\right)$, so $c_{2} \leq 6$. Using Kawamata-Viehweg vanishing theorem and Riemann-Roch formula on $\mathbf{P}^{2}$ we obtain:

$$
\Delta(Y, A)=\operatorname{dim} Y+A^{3}-h^{0}(Y, A)=3+\left(7-c_{2}\right)-\left(9-c_{2}\right)=1 .
$$

Now from the sectional genus formula (cf. [Fuj])

$$
2 g(X, D)-2=\left(K_{X}+2 D\right) D^{2}=0 .
$$

Naturally $g(Y, A)=g(X, D)=1$ for $\chi(Y, t A)=h^{0}(Y, t A)=h^{0}(X, t D)=$ $\chi(X, t D), t \gg 0$.

Observe that $Y$ has rational singularities, so it is locally CohenMacaulay. Therefore $(Y, A)$ is a Del Pezzo variety by [Fuj, theorem 1.6.5]. In particular $B s|A|=\emptyset$ for $c_{2} \leq 5$ (see [Fuj, 1.6.2]) and $\mathcal{E}(2)$ is globally generated. This will be used for the description of $\mathcal{E}$ if $c_{2}=4$ or 5 .

Now assume that $H^{0}(\mathcal{E}(-1)) \neq 0$ and let $s$ be a non-zero section of $\mathcal{E}(-1), Z=\{s=0\}$. If $Z \neq \emptyset$, then for a line intersecting $Z$ in finitely many points:

$$
\left.\mathcal{E}(-1)\right|_{L}=\mathcal{O}(d) \oplus \mathcal{O}(e) \quad d \geq 1, d+e=-3
$$

in contradiction to the ampleness of $\mathcal{E}(3)$. Hence $Z=\emptyset$ and $\mathcal{E} \simeq \mathcal{O}(1) \oplus$ $\mathcal{O}(-2)$.

3.2.1. Now let us assume that $H^{0}(\mathcal{E}(-1))=0, H^{0}(\mathcal{E}) \neq 0$ and set $s \in H^{0}(\mathcal{E}), Z=\{s=0\}$. We consider the following cases:

3.2.1.1. $Z=\emptyset \quad$ Then $\mathcal{E} \simeq \mathcal{O} \oplus \mathcal{O}(-1)$. 
3.2.1.2. $Z$ consists of one point. In that case section $s$ gives rise to the sequence

$$
0 \longrightarrow \mathcal{O} \longrightarrow \mathcal{E} \longrightarrow J_{x}(-1) \longrightarrow 0 .
$$

Observe that $h^{1}(\mathcal{E}(1))=0, h^{1}(\mathcal{E})=1$ and $h^{2}(\mathcal{E})=0$, so there exists exactly one non-trivial extension $\mathcal{F} \in \operatorname{Ext}^{1}(\mathcal{E}(2), \mathcal{O}(-1)) \simeq H^{1}(\mathcal{E})^{\prime}$ of $\mathcal{E}(2)$ by $\mathcal{O}(-1)$. Hence $H^{2}(\mathcal{F}(-2))=0, H^{1}(\mathcal{F}(-1))=0$ and by the CastelnuovoMumford criterion $\mathcal{F}$ is globally generated and it is a Fano bundle on $\mathbf{P}^{2}$. From [SzW3] it follows that $\mathcal{F} \simeq \mathcal{O}^{2} \oplus \mathcal{O}(2)$.

3.2.1.3. $Z$ contains at least 2 points. We consider a line $L$ containing two points from $Z$. Of course $L$ is not contained in $Z$ for $H^{0}(\mathcal{E}(-1))=0$. Therefore:

$$
\left.\mathcal{E}\right|_{L}=\mathcal{O}(d) \oplus \mathcal{O}(e) \quad d \geq 2, d+e=-1
$$

so $\mathcal{E}(3)$ is not ample, a contradiction.

Remark. In fact we proved here that if $\mathcal{E}(3)$ is ample then $\mathcal{E}$ is of the form $1,2,3$ from the theorem or $\mathcal{E}$ is stable (so $c_{2}>0$ ). We will use this remark in section 6 .

From now on we can assume that $c_{2} \geq 1, H^{0}(\mathcal{E}(k))=0$ for $k \leq 0$ and from Serre duality: $H^{2}(\mathcal{E}(k))=0$ for $k \geq-2$.

3.2.2. $c_{2}=1,2,3 \quad$ Since $\chi(\mathcal{E}(1))=4-c_{2}>0$ we have $H^{0}(\mathcal{E}(1)) \neq 0$. Moreover for $\mathcal{E}(2)$ is nef, the order of a jumping line $r$ is $\leq 1$ (according to Hartshorne's notation [SRS1, p. 141]). From theorem 6.2, [ibid.] we find $H^{1}(\mathcal{E}(k))=0$ for $k \geq 1$.

3.2.2.1. $\quad c_{2}=1$ By the Riemann-Roch formula $h^{1}(\mathcal{E})=\chi(\mathcal{E})=0$ and $h^{2}(\mathcal{E}(-1))=0$. So by the Castelnuovo-Mumford theorem $\mathcal{E}(1)$ is globally generated and $\mathcal{E}$ is a Fano sheaf. Now it is easy to see that $\mathcal{E} \simeq T_{\mathbf{P}^{2}}(-2)$, e.g., using the Beilinson spectral sequence cf. [Be] or [OSS, $I I$, theorem 3.1.3] (alternatively one can check that $h^{0}(\mathcal{E}(1))=3$, so the kernel of natural surjection $\mathcal{O}^{3} \rightarrow \mathcal{E}$ is a line bundle, which is completely determined by its first Chern class; by Euler's sequence we get the required isomorphism).

3.2.2.2. $c_{2}=2 \quad$ Then $h^{1}(\mathcal{E})=1, h^{0}(\mathcal{E}(1))=2, h^{0}(\mathcal{E}(2))=7$. Now we use the Beilinson spectral sequence for $\mathcal{E}(2)$ : 


\begin{tabular}{|c|c|c|}
\hline 0 & 0 & 0 \\
$\mathcal{O}(-1)$ & 0 & 0 \\
0 & $\Omega(1)^{2}$ & $\mathcal{O}^{7}$ \\
\hline $\mathcal{E}$ & $\mathcal{E}(1)$ & $\mathcal{E}(2)$ \\
\hline
\end{tabular}

So we obtained the following exact sequence:

$$
0 \longrightarrow \mathcal{O}(-1) \longrightarrow \mathcal{F} \longrightarrow \mathcal{E}(2) \longrightarrow 0
$$

Here $\mathcal{F}=\mathcal{O}^{7} / \Omega(1)^{2}$ is a rank 3 globally generated bundle with Chern classes $c_{1}(\mathcal{F})=2, c_{2}(\mathcal{F})=1$. Therefore $\mathcal{F}$ is a Fano bundle and $\mathcal{F} \simeq \mathcal{O}(1)^{2} \oplus \mathcal{O}$ (see $[\mathrm{SzW} 3]$ ). We have case 5 of the theorem.

3.2.2.3. $c_{2}=3$ Similarly as in 3.2.2.2 we obtain 6 from the theorem.

3.2.3. $c_{2}=4$

3.2.3.1. If $h^{0}(\mathcal{E}(1))=0$, then by the same method as before we obtain the sequence

$$
0 \longrightarrow \mathcal{O}(-1)^{3} \longrightarrow \mathcal{O}^{5} \longrightarrow \mathcal{E}(2) \longrightarrow 0 \text {. }
$$

3.2.3.2. Now assume that $h^{0}(\mathcal{E}(1)) \neq 0$. In 3.2 .0 we proved that $\mathcal{E}(2)$ is globally generated. Now let us consider the kernel $\mathcal{F}$ of evaluation $\mathcal{O}^{5} \rightarrow$ $\mathcal{E}(2)$. Naturally $\mathcal{F}^{*}$ is a rank 3 globally generated vector bundle on $\mathbf{P}^{2}$, so by Serre lemma ([OSS, I, 4.3.1]) we have an exact sequence:

$$
0 \longrightarrow \mathcal{O} \longrightarrow \mathcal{F}^{*} \longrightarrow \mathcal{G}(2) \longrightarrow 0
$$

where $\mathcal{G}$ is a rank 2 vector bundle with Chern classes $c_{1}(\mathcal{G})=-1, c_{2}(\mathcal{G})=1$. In this case $\mathcal{G}$ is a weak Fano sheaf, for $\mathcal{G}(2)$ is globally generated. Therefore from 3.2.2.1 it follows that $\mathcal{G} \simeq T_{\mathbf{P}^{2}}(-2)$. Because $\operatorname{dim} \operatorname{Ext}^{1}(\mathcal{G}(2), \mathcal{O})=$ $\operatorname{dim} H^{1}(\mathcal{G}(-1))^{\prime}=\operatorname{dim} H^{1}\left(T_{\mathbf{P}^{2}}(-3)\right)=1$ we have $\mathcal{F}^{*} \simeq \mathcal{O} \oplus T_{\mathbf{P}^{2}}$ or $\mathcal{F}^{*} \simeq$ $\mathcal{O}(2) \oplus T_{\mathbf{P}^{2}}(-1)$. But the first case cannot happen for $H^{0}(\mathcal{F})=0$, so $\mathcal{F} \simeq \Omega(1) \oplus \mathcal{O}(-2)$ and we have an exact sequence:

$$
0 \longrightarrow \mathcal{O}(-2) \longrightarrow \mathcal{O}^{5} / \Omega(1) \longrightarrow \mathcal{E}(2) \longrightarrow 0
$$

Applying the classification of Fano bundles on $\mathbf{P}^{2}$ we get $\mathcal{O}^{5} / \Omega(1) \simeq \mathcal{O}(1) \oplus$ $\mathcal{O}^{2}$. 
3.2.4. $c_{2}=5$ Then $h^{0}(\mathcal{E}(2))=4$ and $h^{0}(\mathcal{E}(1))=0$ by the criterion of Nakai-Moishezon $\left((\xi+H) D^{2}=4-c_{2}<0\right)$. Now we already know that the bundle $\mathcal{E}(2)$ is globally generated, so for some bundle $\mathcal{F}$ we get the sequence

$$
0 \longrightarrow \mathcal{F}(-2) \longrightarrow \mathcal{O}^{4} \longrightarrow \mathcal{E}(2) \longrightarrow 0 \text {. }
$$

As before $\mathcal{F}^{*}$ is weak Fano and $\mathcal{F}^{*} \simeq \mathcal{O} \oplus \mathcal{O}(-1)$.

3.2.5. $c_{2}=6$ We will prove that this case cannot occur. On the contrary assume it can happen. In 3.2 .0 we proved that $\phi: X \rightarrow Y$ is a crepant resolution of Del Pezzo variety of degree 1 (i.e., $A^{3}=1$ ). In that case the base scheme of $|A|$ is a smooth point $y$ (cf. [Fuj, 1.6.14]). Therefore the base scheme of $|D|$ is also a point $x$ (the preimage of $y$ under $\phi$ ). From this one can easily see that $\mathcal{E}$ fits into the following exact sequence:

$$
0 \rightarrow \mathcal{O}_{\mathbf{P}^{2}}(-3) \stackrel{i}{\longrightarrow} \mathcal{O}_{\mathbf{P}^{2}}^{3} \longrightarrow \mathcal{E}(2) \longrightarrow \mathcal{O}_{P} \rightarrow 0,
$$

where $P=p(x)$. The inclusion $i$ is given by three forms $\phi_{0}, \phi_{1}, \phi_{2}$, of degree 3 vanishing at $P$.

If we blow up $X$ at the point $x$ we obtain a smooth manifold $Z$, which is a divisor in $\mathbf{P}^{2} \times \mathbf{P}^{2}$ given by the equation

$$
\begin{array}{r}
\phi\left(x_{0}, x_{1}, x_{2} ; y_{0}, y_{1}, y_{2}\right) \\
\quad=\phi_{0}\left(x_{0}, x_{1}, x_{2}\right) y_{0}+\phi_{1}\left(x_{0}, x_{1}, x_{2}\right) y_{1}+\phi_{2}\left(x_{0}, x_{1}, x_{2}\right) y_{2}=0
\end{array}
$$

where $\left[x_{0}, x_{1}, x_{2}\right]$ are homogeneous coordinates in the first copy of $\mathbf{P}^{2}$, and $\left[y_{0}, y_{1}, y_{2}\right]$ in the second one. We will prove that there are no smooth manifolds in $\mathbf{P}^{2} \times \mathbf{P}^{2}$ satisfying such equation for any forms $\phi_{i}$ having one common zero in $\mathbf{P}^{2}$.

Without loss of generality we can assume that $P=[0,0,1]$. The singular locus of $Z$ is given by the following system of equations:

$$
\left\{\begin{aligned}
\phi\left(x_{0}, x_{1}, x_{2} ; y_{0}, y_{1}, y_{2}\right) & =0 \\
\frac{\partial \phi}{\partial x_{i}}\left(x_{0}, x_{1}, x_{2} ; y_{0}, y_{1}, y_{2}\right) & =0 \\
\frac{\partial \phi}{\partial y_{i}}\left(x_{0}, x_{1}, x_{2} ; y_{0}, y_{1}, y_{2}\right) & =\phi_{i}\left(x_{0}, x_{1}, x_{2}\right)=0
\end{aligned}\right.
$$

for $i=0,1,2$. From these equations we infer that $\left[x_{0}, x_{1}, x_{2}\right]=[0,0,1]$, and the system is equivalent to

$$
\sum_{j=0}^{2} \frac{\partial \phi_{j}}{\partial x_{i}}(0,0,1) y_{j}=0 \quad \text { for } i=0,1,2
$$


But $\frac{\partial \phi_{j}}{\partial x_{2}}(0,0,1)=0$, so this system has always a non-zero solution. In particular $Z$ has a singular point on the exceptional divisor of the blow up, a contradiction.

This completes the proof of our theorem.

COROLlaRY 3.3. Let $\mathcal{F}$ be a globally generated bundle of rank 2 on $\mathbf{P}^{2}$ with the first Chern class $c_{1}(\mathcal{F})=3$. Let us denote $\mathcal{E}=\mathcal{F}(-2)$. Then $\mathcal{E}$ is of the form 1-9 from theorem 3.2 or $c_{2}(\mathcal{E})=7$ and $\mathcal{E}$ can be put in the form:

$$
0 \longrightarrow \mathcal{O}(-3) \longrightarrow \mathcal{O}^{3} \longrightarrow \mathcal{E}(2) \longrightarrow 0
$$

Proof. At first let us remark that $-K_{\mathbf{P}(\mathcal{F})}=2 \xi_{\mathcal{F}}$ is nef because $\mathcal{F}$ is globally generated. It follows that

$$
\left(-K_{\mathbf{P}(\mathcal{F})}\right)^{3}=8\left(7-c_{2}(\mathcal{E})\right) \geq 0 .
$$

If $c_{2}(\mathcal{E}) \leq 6$, then theorem 3.2 implies that $\mathcal{E}$ is a bundle of the form $1-$ 9 , theorem 3.2. So we can assume $c_{2}(\mathcal{E})=7$. As in 3.2 .4 we prove that $h^{0}(\mathcal{E}(1))=0$. Because $\mathcal{E}(2)$ is globally generated so the maximal rank of the jumping line $r \leq 1$ (see 3.2.2). Using [SRS1, theorem 6.2] we obtain $h^{1}(\mathcal{E}(2)) \leq 1$. Now the Riemann-Roch theorem implies:

$$
h^{0}(\mathcal{E}(2)) \leq \chi(\mathcal{E}(2))+h^{1}(\mathcal{E}(2)) \leq 3
$$

From this it can be easily seen that $\mathcal{E}$ is of the form 3.3.1.

Remark. Using corollary 3.3 one can easily describe globally generated bundles of rank $r>2$ with $c_{1}=3$ on $\mathbf{P}^{2}$. The structure of such bundles can be described using [SzW3, lemma 3.1]; all of them are extensions of the bundles of the same type but of the lower rank. Unfortunately there are already 38 such bundles of rank 3 .

\section{$\S 4$. Divisorial contractions}

THEOREM 4.1. If $\phi: X \rightarrow Y$ is a divisorial contraction, $X$ is smooth, $E$ is an exepctional divisor of $\phi, \operatorname{dim} \phi(E)=\operatorname{dim} E-1, C$ is a general fiber of $\left.\phi\right|_{E}: E \rightarrow \phi(E)$, then $C \simeq \mathbf{P}^{1}$ and $C \cdot E=-1, K_{X} \cdot C=-1$. Moreover if $X$ is Fano and $\rho(X)=2$, then $-K_{X}+E$ is a good supporting divisor for $\phi$. 
Proof. This is an immediate consequence of [A, theorem 2.1]: there exists a Cartier divisor $L$ on $X$ such that $\left(C,\left.L\right|_{C}\right) \simeq\left(\mathbf{P}^{1}, \mathcal{O}_{\mathbf{P}^{1}}(1)\right)$ and $\omega_{C}=\mathcal{O}_{C}((-p-q) L)$, where $\mathcal{O}_{C}\left(-K_{X}\right) \simeq \mathcal{O}_{C}(p L)$ and $\mathcal{O}_{C}(-E) \simeq \mathcal{O}_{C}(q L)$, $p, q \geq 1$. Since $\omega_{\mathbf{P}^{1}} \simeq \mathcal{O}_{\mathbf{P}^{1}}(-2), p=q=1$ and we have the first part of theorem. The other one is obvious for $\left(-K_{X}+E\right) \cdot C=0$.

Remark. The following more general theorem is also valid: if $C$ is a one-dimensional fiber, then in some neighborhood of $C, \phi$ is the blow down of smooth divisor $E$ to a smooth subvariety of $Y$ (cf. [A, theorem 2.3] and [AW1]). But we will use only this simple version of this theorem.

TheOREM 4.2. Let $\mathcal{E}$ be a rank $r \geq 2$ Fano sheaf on $\mathbf{P}^{n}, n \geq 2$ and assume that the "other" contraction $\phi: X=\mathbf{P}(\mathcal{E}) \rightarrow Y$ is divisorial, $E, C$ are as above. Then $k=H \cdot C$ is positive and divides $\left(\begin{array}{c}r+n-2 \\ n\end{array}\right)$. In particular for $r=2$ it follows that $H \cdot C=1$ and $-K_{X}-H$ is a good supporting divisor for $\phi$.

Proof. We have the following equalities:

$$
\begin{aligned}
-K_{X} & =r \xi+\left(n+1-c_{1}\right) H, & & c_{1}=c_{1}(\mathcal{E}) \\
E & =a \xi+b H, & & a, b \in Z .
\end{aligned}
$$

If we apply theorem 4.1 we obtain:

$$
\begin{aligned}
-K_{X} \cdot C & =1 \\
E \cdot C & =-1
\end{aligned}
$$

for general fiber $C$ of morphism $\left.\phi\right|_{E}$. Let $k=H \cdot C>0$. We set $D_{R}:=$ $-K_{X}-\frac{1}{k} H$. This is a good supporting $\mathbf{Q}$-divisor for $\phi$, because $D_{R} \cdot C=0$. One can also see that $E+\frac{1}{k} H$ is also a good supporting divisor for $\phi$. Therefore comparing coefficients with $\xi$, we get

$$
E+\frac{1}{k} H=\frac{a}{r} D_{R}
$$

Now if we compare coefficients with $H$ we get:

$$
b=\frac{a}{r}\left(n+1-c_{1}-\frac{1}{k}\right)-\frac{1}{k} \in \mathbf{Z} .
$$

Hence $k$ divides $\frac{a+r}{(a, r)}=-k\left[\frac{r}{(a, r)} b-\frac{a}{(a, r)}\left(n+1-c_{1}\right)\right]$, where $(a, r)$ stands for the greatest common divisor of $a$ and $r$. Naturally we have also the 
following equalities: $H^{n+1} \equiv 0$ and $H^{n} \xi^{r-1}=1$. Moreover we know that 1-cycle $D_{R}^{r+n-2} E$ is numerically trivial. Hence by (4.2.1) we get:

$$
\frac{a}{r} D_{R}^{r+n-1}=\frac{1}{k} H D_{R}^{r+n-2}
$$

If we multiply both sides of (4.2.3) by $\frac{k^{n}}{r^{r-1}}$ and substitute $D_{R}=r \xi+(n+$ $\left.1-c_{1}-\frac{1}{k}\right) H$, we get:

$$
\begin{aligned}
\frac{a}{r}\left(\xi^{r-1} H^{n}\left(\begin{array}{c}
r+n-1 \\
n
\end{array}\right)\left(\left(n+1-c_{1}\right) k-1\right)^{n}\right) \\
\quad+\left(\text { the sum of } \xi^{p} H^{q} \text { with integral, divisible by } k, \text { coefficients }\right) \\
=\xi^{r-1} H^{n}\left(\begin{array}{c}
r+n-2 \\
n-1
\end{array}\right) \cdot\left(\left(n+1-c_{1}\right) k-1\right)^{n-1} \\
\quad+\left(\text { the sum of } 2 \xi^{p} H^{q} \text { with integral, divisible by } k \text { coefficients }\right) .
\end{aligned}
$$

From this equality it follows that $\frac{r}{(a, r)}$ divides $\left(\begin{array}{c}r+n-1 \\ n\end{array}\right)\left(\left(n+1-c_{1}\right) k-1\right)^{n}$. Now we can choose $r_{1}$ and $r_{2}$ such that: $r_{1} r_{2}=\frac{r}{(a, r)}, r_{1}$ divides $((n+1-$ $\left.\left.c_{1}\right) k-1\right)^{n}$ and $r_{2}$ divides $\left(\begin{array}{c}r+n-1 \\ n\end{array}\right)$. Then

$$
\frac{a}{(a, r) r_{2}}\left(\begin{array}{c}
r+n-1 \\
n
\end{array}\right)(-1)^{n} \equiv r_{1}\left(\begin{array}{c}
r+n-2 \\
n-1
\end{array}\right)(-1)^{n-1} \quad(\bmod k)
$$

so

$$
\frac{a+r}{(a, r)} \frac{\left(\begin{array}{c}
r+n-1 \\
n
\end{array}\right)}{r_{2}} \equiv r_{1}\left(\left(\begin{array}{c}
r+n-1 \\
n
\end{array}\right)-\left(\begin{array}{c}
r+n-2 \\
n-1
\end{array}\right)\right) \quad(\bmod k) .
$$

Because $k$ divides $\frac{a+r}{(a, r)}$ and $\left(r_{1}, k\right)=1$ it follows that $k$ divides $\left(\begin{array}{c}r+n-2 \\ n\end{array}\right)$. The last statement of the theorem is obvious for $k$ must be equal to 1 and $D_{R}=-K_{X}-H$,

From now on in the next two sections we will consider the following situation: $\mathcal{E}$ is a rank 2 Fano sheaf on $\mathbf{P}^{3}$, normalized in such a way that $c_{1}=0$ or -1 and $\phi: X=\mathbf{P}(\mathcal{E}) \rightarrow Y$ is the "other" contraction. In this section we will assume in addition that $\phi$ is divisorial. Let $E$ denotes the exceptional divisor for $\phi$.

Lemma 4.3. Let $\mathcal{E}$ be as above. Then $c_{3}=0, c_{2} \leq 1$ or the Chern classes of the sheaf $\mathcal{E}$ are of one of the following types: $(-1,2,2),(-1,3,5)$, $(-1,3,7),(-1,4,10),(-1,4,12),(-1,5,17),(0,1,2),(-1,1,3)$. 
Proof.

4.3.1. Let us assume that $\operatorname{dim} \phi(E)=2$. First observe that $c_{1}(\mathcal{E})=$ -1 , because otherwise $\mathbf{P}(\mathcal{E})$ would be a Fano 4 -fold of index 2 and then this type of contraction can not occur. Next, applying theorem 4.2 we get that $-K_{X}-H=2(\xi+2 H)$ is a good supporting divisor for $\phi$. Then by (4.2.2) we know that $b=2 a-1$ and by (4.2.3):

$$
a(\xi+2 H)^{4}=H(\xi+2 H)^{3} .
$$

It is easy to see that $a>0$ for otherwise $-H=E+(-a)(\xi+2 H)$ is effective. Since $\phi$ is divisorial:

$$
\begin{gathered}
0<(\xi+2 H)^{4}=15-6 c_{2}+c_{3} \\
0<H(\xi+2 H)^{3}=7-c_{2}
\end{gathered}
$$

and from the above equalities:

$$
a\left(15-6 c_{2}+c_{3}\right)=7-c_{2}
$$

Now we claim that $2 \leq c_{2} \leq 6$ : this is straightforward consequence of (4.3.1.1). Besides $c_{1} c_{2} \equiv c_{3} \quad(\bmod 2)($ see $[\mathrm{OSS}])$ and $\left.\mathcal{E}\right|_{\mathbf{P}^{2}}$ is weak Fano. If we take it into account we obtain the following possibilities:

a) $c_{2}=2, c_{3}=2, a=1$

b) $c_{2}=3, c_{3}=5, a=2$ or $c_{3}=7, a=1$

c) $c_{2}=4, c_{3}=10, a=3$ or $c_{3}=12, a=1$

d) $c_{2}=5, c_{3}=17, a=1$

4.3.2. Now assume that $\operatorname{dim} \phi(E) \leq 1$. Then the 1 -cycle $D_{R}^{2} E$ is numerically trivial. $D_{R}$ and $E$ are positive multiples of $(\xi+u H)$ and $(\xi+$ $v H)$, respectively, where $u, v \in \mathbf{Q}$. So we have the following equalities:

$$
\begin{aligned}
0 & =(\xi+u H)^{2}(\xi+v H) H \\
& =c_{1}{ }^{2}-c_{2}+2 c_{1} u+u^{2}+\left(c_{1}+2 u\right) v \\
0 & =(\xi+u H)^{2}(\xi+v H) \xi \\
& =c_{1}{ }^{3}-2 c_{1} c_{2}+c_{3}+2\left(c_{1}{ }^{2}-c_{2}\right) u+c_{1} u^{2}+\left(c_{1}{ }^{2}-c_{2}+2 c_{1} u+u^{2}\right) v .
\end{aligned}
$$


Comparing these two formulas we get the following equality, involving only $c_{1}, c_{2}, c_{3}$ and $u$ :

$$
\begin{aligned}
& \left(c_{1}{ }^{2}-c_{2}+2 c_{1} u+u^{2}\right)^{2} \\
& \quad-\left(c_{1}+2 u\right)\left(c_{1}{ }^{3}-2 c_{1} c_{2}+c_{3}+2\left(c_{1}{ }^{2}-c_{2}\right) u+c_{1} u^{2}\right)=0
\end{aligned}
$$

Therefore $u$ is an integer (for $\mathbf{Z}$ is integrally closed in $\mathbf{Q}$ ). Now let us write $-K_{X}$ by means of $D_{R}$ and $H$ :

$$
-K_{X}=2(\xi+2 H)+\left(4-c_{1}-2 u\right) H
$$

For $-K_{X}$ is ample, so $4-c_{1}-2 u>0$ and by lemma 2.7 we have the following possibilities:

a) if $c_{1}=0$, then $u=0$ or $u=1$

b) if $c_{1}=-1$, then $u=1$ or $u=2$

We will consider each of these cases separately, taking into account the following inequality:

$$
D_{R}^{4}=(\xi+u H)^{4}>0
$$

4.3.2.3. $\left(c_{1}=0, u=0\right) \quad$ Then by (4.3.2.1): $c_{2}=0, c_{3}=0$, but $\xi^{4}=0$, which contradicts (4.3.2.2).

4.3.2.4. $\left(c_{1}=0, u=1\right) \quad$ By (4.3.2.1): $2 c_{3}=\left(1+c_{2}\right)^{2}$. Then $\xi+H$ is nef, so $\mathbf{P}\left(\left.\mathcal{E}\right|_{\mathbf{P}^{2}}\right)$ for a general plane is Fano. Therefore by [SzW2] we have the following possibilities:

a) $c_{2}=-1, c_{3}=0,(\xi+H)^{4}=c_{3}-4 c_{2}+4=8$

b) $c_{2}=1, c_{3}=2,(\xi+H)^{4}=2$

c) $c_{2}=3, c_{3}=8,(\xi+H)^{4}=0$ - in a contradiction to (4.3.2.2).

4.3.2.5. $\left(c_{1}=0, u=1\right) \quad$ By (4.3.2.1): $2 c_{3}=c_{2}^{2}$ Then $\mathbf{P}\left(\left.\mathcal{E}\right|_{\mathbf{P}^{2}}\right)$ is Fano and by Nakai-Moishezon criterion: $(\xi+H)^{3} H=1-c_{2}>0$, so $c_{2} \leq 0$. As above the only case is:

$$
c_{2}=0, \quad c_{3}=0, \quad(\xi+H)^{4}=1
$$

4.3.2.6. $\left(c_{1}=-1, u=2\right) \quad$ By (4.3.2.1): $3 c_{3}=\left(c_{2}+2\right)^{2}, \mathbf{P}\left(\left.\mathcal{E}\right|_{\mathbf{P}^{2}}\right)$ is weak Fano, so by theorem 3.2 we get: 
a) $c_{2}=-2, c_{3}=0$,

b) $c_{2}=1, c_{3}=3$,

c) $c_{2}=4, c_{3}=12$.

TheOREM 4.4. A classification of a rank 2 Fano sheaves $\mathcal{E}$ on $\mathbf{P}^{3}$ with a divisorial contraction:

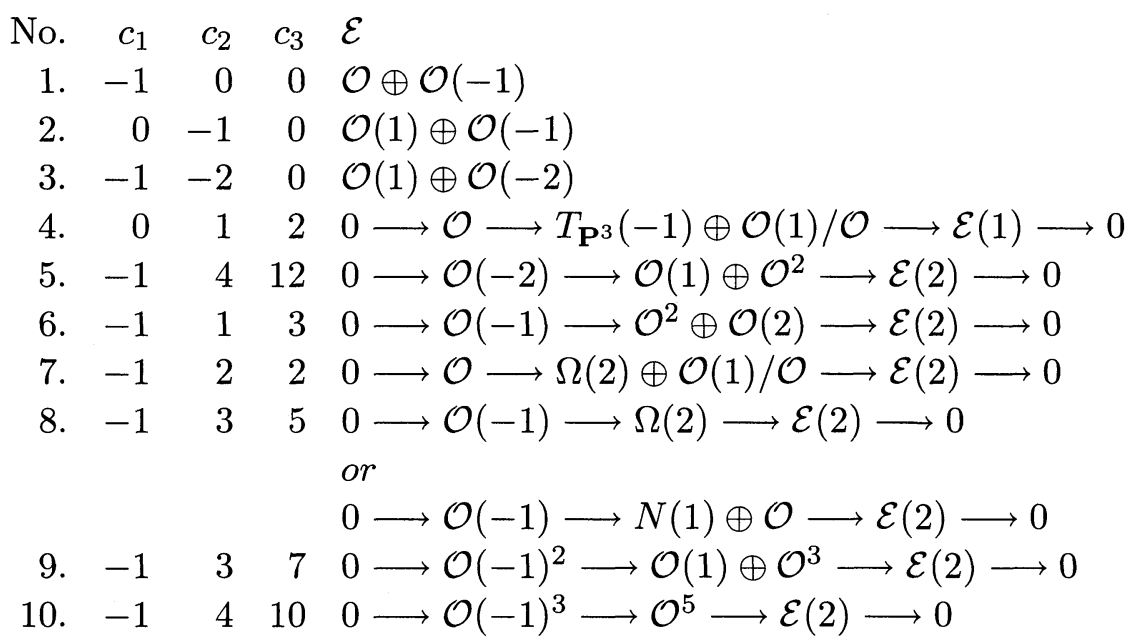

Proof.

Remark. Proofs of theorems 4.4, 5.2 and 6.2 are not independent: we prove only that if the contraction is divisorial (respectively: fiber, small), then there are only the following possibilities. The fact that the contraction of the sheaf is exactly of such type as we suppose follows from computations - we obtain either different Chern classes or this can be easily seen.

First note that there does not exist Fano sheaf with Chern classes $(-1,5,17)$ (we use notation: $\left.\left(c_{1}, c_{2}, c_{3}\right)\right)$. It can be easily seen that $h^{0}(\mathcal{E}(1))$ $=\chi(\mathcal{E}(1))=1 / 2\left(c_{3}+10-5 c_{2}\right)=1$. But from the divisorial sequence:

$$
\left.0 \longrightarrow \mathcal{E} \longrightarrow \mathcal{E}(1) \longrightarrow \mathcal{E}(1)\right|_{\mathbf{P}^{2}} \longrightarrow 0
$$

and from theorem 3.2 it follows that $h^{0}(\mathcal{E}(1))=0$, a contradiction.

Cases $c_{3}=0, c_{2} \leq 1$ are simple and done in [SzW1] (one can also obtain them by restriction to $\mathbf{P}^{2}$ ). Next, we consider remaining cases: 
4.4.2. $(0,1,2) \quad$ By fact 1.2 .4 one should only prove that $\mathcal{E}(1)$ extends (which is the case if $H^{2}\left((\mathcal{E}(1))^{*}\right)=0$ ) and use the classification of numerically effective bundles with first Chern class equal to 2, see [PSW1]. From a long exact cohomology sequence for (4.4.1) twisted by $\mathcal{O}(-1)$ we get:

$$
H^{1}\left(\left.\mathcal{E}\right|_{\mathbf{P}^{2}}\right) \longrightarrow H^{2}(\mathcal{E}(-1))=H^{2}\left((\mathcal{E}(1))^{*}\right) \longrightarrow H^{2}(\mathcal{E})=0
$$

Since $\left.\mathcal{E}\right|_{\mathbf{p}^{2}}$ is Fano bundle: $H^{1}\left(\left.\mathcal{E}\right|_{\mathbf{P}^{2}}\right)=0$.

In the remaining cases the sheaf $\left.\mathcal{E}\right|_{\mathbf{P}^{2}}$ is a weak Fano, since $\mathcal{E}(2)$ is nef by theorem 4.2 .

4.4.3. (-1,4,12) Applying (4.4.1), theorem 3.2 and lemma 2.4.1 we know that there exist non-trivial extension $\mathcal{F}$ :

$$
0 \longrightarrow \mathcal{O}(-2) \longrightarrow \mathcal{F} \longrightarrow \mathcal{E}(2) \longrightarrow 0
$$

such that $\mathcal{F} \simeq \mathcal{O}(1) \oplus \mathcal{O}^{2}$.

4.4.4. $(-1,1,3)$ We know $\left.\mathcal{E}\right|_{P^{2}}$ and by Serre construction:

$$
0 \longrightarrow \mathcal{O} \longrightarrow \mathcal{E} \longrightarrow J_{L}(-1) \longrightarrow 0
$$

where $L$ is a line in $\mathbf{P}^{3}$. Now we can use lemma 2.4.1 to get a non-trivial extension $\mathcal{F}$ :

$$
0 \longrightarrow \mathcal{O}(-1) \longrightarrow \mathcal{F} \longrightarrow \mathcal{E}(2) \longrightarrow 0
$$

where $\mathcal{F} \simeq \mathcal{O}(2) \oplus \mathcal{O}^{2}$. This gives case 6 of our theorem.

In the remaining cases we compute cohomology of $\mathcal{E}$ using known cohomology of $\left.\mathcal{E}\right|_{\mathbf{P}^{2}}$, divisorial sequence and the Riemann-Roch formula for $\mathcal{E}(-1), \ldots, \mathcal{E}(2)$.

4.4.5. $(-1,2,2) \quad$ Observe first that $H^{2}\left((\mathcal{E}(2))^{*}\right)=H^{2}(\mathcal{E}(-1))=0$, so by fact 1.2.4 there exists non-trivial extension $\mathcal{F}$ of $\mathcal{E}(2)$ by $\mathcal{O}$, where $\mathcal{F}$ is locally free. From cohomology exact sequence we get:

$$
H^{2}(\mathcal{E}(-2)) \stackrel{\delta}{\longrightarrow} H^{3}(\mathcal{O}(-4)) \longrightarrow H^{3}(\mathcal{F}(-4)) \longrightarrow H^{3}(\mathcal{E}(-2))=0,
$$

so by non-triviality of $\delta: H^{3}(\mathcal{F}(-4))=0$. One can compute the rest of cohomology of $\mathcal{F}(-k)(k=1,2,3,4)$ using cohomology of $\mathcal{E}(-k+2)$. In particular $\operatorname{dim} \operatorname{Ext}^{1}(\mathcal{F}, \mathcal{O})=h^{2}(\mathcal{F}(-4))=1$. So there exists a non-trivial extension $\mathcal{G}$ :

$$
0 \longrightarrow \mathcal{O} \longrightarrow \mathcal{G} \longrightarrow \mathcal{F} \longrightarrow 0
$$


Computing cohomology of sheaves $\mathcal{G}(k)$ for $k=-1, \ldots-4$ and using the Beilinson spectral sequence for $\mathcal{G}(-1)$ we get:

$$
0 \longrightarrow \mathcal{O}(1) \longrightarrow \mathcal{G} \longrightarrow \Omega(2) \longrightarrow 0
$$

Now because $\operatorname{Ext}^{1}(\Omega(2), \mathcal{O}(1))=0$, so $\mathcal{G} \simeq \Omega(2) \oplus \mathcal{O}(1)$ and we get case 7 .

4.4.6. $(-1,3,5)$ From the sequence

$$
\begin{aligned}
0=H^{0}\left(\left.\mathcal{E}\right|_{\mathbf{P}^{2}}\right) & \longrightarrow H^{1}(\mathcal{E}(-1)) \longrightarrow H^{1}(\mathcal{E}) \\
& \longrightarrow H^{1}\left(\left.\mathcal{E}\right|_{\mathbf{P}^{2}}\right) \longrightarrow H^{2}(\mathcal{E}(-1)) \longrightarrow H^{2}(\mathcal{E})=0
\end{aligned}
$$

and $h^{1}(\mathcal{E})=1$ we can only deduce, that $h^{1}(\mathcal{E}(-1)) \leq 1$. Suppose for a moment that $h^{1}(\mathcal{E}(-1))=1$. Then $h^{2}(\mathcal{E}(-1))=2$ and from the Beilinson spectral sequence:

$$
0 \longrightarrow \mathcal{O}(-1)^{2} \longrightarrow \mathcal{F} \longrightarrow \mathcal{E}(2) \longrightarrow 0
$$

for some globally generated sheaf $\mathcal{F}$ with $c_{1}(\mathcal{F})=1, c_{2}(\mathcal{F})=0$. As above $\mathcal{F} \simeq \mathcal{O}^{3} \oplus \mathcal{O}(1)$ in contradiction to $c_{3}(\mathcal{E})=7$. So we proved that $h^{1}(\mathcal{E}(-1))=0, h^{2}(\mathcal{E}(-1))=1$ and from Beilinson spectral sequence:

$$
0 \longrightarrow \mathcal{O}(-1) \longrightarrow \mathcal{O}^{6} / T_{\mathbf{P}^{3}}(-2)=\mathcal{F} \longrightarrow \mathcal{E}(2) \longrightarrow 0,
$$

where $\mathcal{F}$ is possibly non-locally free. We claim that in fact this sheaf must be locally free. Applying to twisted (4.4.6.1) a functor Ext we get the dual sequence:

$$
0 \longrightarrow(\mathcal{E}(3))^{*} \longrightarrow(\mathcal{F}(1))^{*} \longrightarrow J_{Y} \longrightarrow 0,
$$

where $Y$ is a finite set of points (a support of $\operatorname{Ext}^{1}(\mathcal{E}(3), \mathcal{O})$ ). Using $\operatorname{Ext}^{1}\left(J_{Y}, \mathcal{O}_{\mathbf{P}^{3}}\right)=0$ (see p. $\left.690,[\mathrm{GH}]\right)$ and dualizing once more we obtain the following canonical diagram with exact rows:

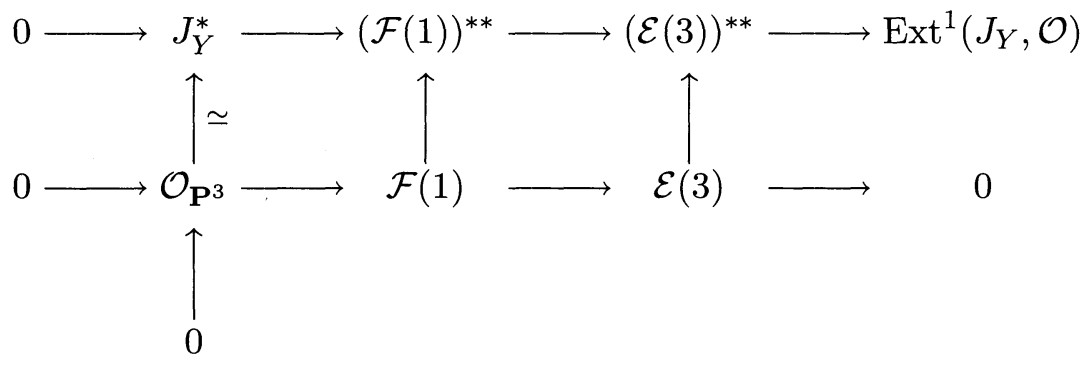


By lemma about 5 isomorphisms $\mathcal{F}$ is reflexive. Let us now consider the sequence:

$$
0 \longrightarrow \mathcal{O} \stackrel{s}{\longrightarrow} \mathcal{F} \longrightarrow \mathcal{G} \longrightarrow 0
$$

For a general section $s$ a sheaf $\mathcal{G}$ is locally free besides finite number of points, where $\mathcal{F}$ is singular. Similarly as above one can easily prove that $\mathcal{G}$ is reflexive, so $\mathcal{G} \simeq N(1)$. Because $\operatorname{Ext}^{1}(N(1), \mathcal{O})=1, \mathcal{F} \simeq N(1) \oplus \mathcal{O}$ or $\mathcal{F} \simeq \Omega(2)$.

4.4.7. $(-1,3,7)$ This can be easily obtained by the use of Beilinson spectral sequence.

4.4.8. $(-1,4,10) \quad$ If $h^{2}(\mathcal{E}) \neq 0$, then as in 4.4 .3 we prove that $\mathcal{E}$ looks like 5 -a contradiction. Hence $h^{2}(\mathcal{E})=0$ and by Beilinson spectral sequence we get case 10 .

Let us remark that in all cases we have an exact sequence of the form:

$$
0 \longrightarrow \mathcal{G} \stackrel{\sigma}{\longrightarrow} \mathcal{F} \longrightarrow \mathcal{E}(2) \longrightarrow 0,
$$

where $\mathcal{F}, \mathcal{G}$ are locally free and the sheaf $\operatorname{Hom}(\mathcal{G}, \mathcal{F})=\mathcal{G}^{*} \otimes \mathcal{F}$ is generated by global sections. Therefore by [BW, lemma 3.1] for generic $\sigma \in$ $\operatorname{Hom}(\mathcal{G}, \mathcal{F}), \mathcal{E}(2)$ is a Bănică sheaf and $\mathbf{P}(\mathcal{E})$ is a smooth Fano variety.

\section{$\S 5$. Fiber contractions}

We use notation compatible with that before theorem 4.2 assuming $\phi$ is a fiber contraction.

Lemma 5.1. Let $\mathcal{E}$ be as above. Then $c_{3}=0, c_{2} \leq 1$ or Chern classes of the sheaf $\mathcal{E}$ are of one of the following types: $(0,2,4),(0,3,8),(-1,3,3)$, $(-1,3,7),(-1,1,1),(-1,5,15),(-1,7,27)$. Moreover $\mathcal{E}(2)$ is nef.

Proof. Let $D_{R}=\xi+u H$ denote a good supporting Q-divisor of $\phi$. Then $D_{R}^{4}=0$ (see $[\mathrm{A}$, lemma 1.4]). Furthermore by Nakai-Moishezon criterion $D_{R}^{3} H \geq 0$. Corollary 2.6 leads now to:

$$
\begin{gathered}
(\xi+u H)^{4}=0 \Leftrightarrow 4 u^{3}+6 c_{1} u^{2}+4\left(c_{1}^{2}-c_{2}\right) u+c_{1}{ }^{3}-2 c_{1} c_{2}+c_{3}=0 \\
(\xi+u H)^{3} H \geq 0 \Leftrightarrow 3 u^{2}+3 c_{1} u+c_{1}^{2}-c_{2} \geq 0
\end{gathered}
$$

By similar arguments as in divisorial case:

$$
-K_{X}=2(\xi+u H)+\left(4-c_{1}-2 u\right) H
$$


where

$$
\left(4-c_{1}-2 u\right) H>0 \text {. }
$$

By (5.1.1), if we write $u=\frac{a}{b},(a, b)=1, a, b \in Z$, then $b \mid 2$. Combining the fact that $c_{2} \geq 0$ or a sheaf is decomposable bundle (by classifications of Fano bundles and weak Fano bundles on $\mathbf{P}^{2}$ ) with lemma 2.7, (5.1.1) and (5.1.2) we get the lemma (the last statement is obvious).

TheORem 5.2. Classification of rank 2 Fano sheaves on $\mathbf{P}^{3}$ with two fiber type contractions:

$$
\begin{array}{lrrrll} 
& c_{1} & c_{2} & c_{3} & \\
1 . & 0 & 0 & 0 & \mathcal{O} \oplus \mathcal{O} \\
\text { 2. } & 0 & 1 & 0 & \mathrm{~N}-\text { null correlation } \\
\text { 3. } & 0 & 2 & 4 & 0 \longrightarrow \mathcal{O}(-1)^{2} \longrightarrow \mathcal{O}^{4} \longrightarrow \mathcal{E}(1) \longrightarrow 0 \\
\text { 4. } & 0 & 3 & 8 & 0 \longrightarrow \mathcal{O}(-2) \longrightarrow \mathcal{O}^{3} \longrightarrow \mathcal{E}(1) \longrightarrow 0 \\
5 . & -1 & 1 & 1 & 0 \longrightarrow \mathcal{O}(-1) \longrightarrow \mathcal{O}^{3} \longrightarrow \mathcal{E}(1) \longrightarrow 0 \\
6 . & -1 & 5 & 15 & 0 \longrightarrow \mathcal{O}(-1) \oplus \mathcal{O}(-2) \longrightarrow \mathcal{O}^{4} \longrightarrow \mathcal{E}(2) \longrightarrow 0 \\
\text { 7. } & -1 & 7 & 27 & 0 \longrightarrow \mathcal{O}(-3) \longrightarrow \mathcal{O}^{3} \longrightarrow \mathcal{E}(2) \longrightarrow 0
\end{array}
$$

Proof. We first exclude cases $(-1,3,3)$ and $(-1,3,7)$. In case $(-1,3,7)$ $\xi+\frac{3}{2} H$ is nef, so $\left.\mathcal{E}\right|_{\mathbf{P}^{2}}$ is Fano-this is impossible by [SzW2]. As for the other case we have the following commutative diagram:

$$
\begin{gathered}
X=\mathbf{P}(\mathcal{E}) \underset{\uparrow}{\longrightarrow} Y \\
X^{\prime}=\mathbf{P}\left(\left.\mathcal{E}\right|_{\mathbf{P}^{2}}\right)
\end{gathered}
$$

Clearly on $Y$ there exists an ample divisor $A$ such that: $\phi^{*} A=\xi+2 H$ and $\xi+\left.2 H\right|_{X^{\prime}}=\left(\phi^{\prime}\right)^{*} A$. Hence we get: $h^{0}(Y, A)=h^{0}\left(\mathbf{P}^{2},\left.\mathcal{E}(2)\right|_{\mathbf{P}^{2}}\right)=$ $h^{0}\left(\mathbf{P}^{3}, \mathcal{E}(2)\right)$. On the other hand: $h^{0}\left(\mathbf{P}^{2},\left.\mathcal{E}(2)\right|_{\mathbf{P}^{2}}\right)=6$ and $h^{0}\left(\mathbf{P}^{3}, \mathcal{E}(2)\right)=$ $\chi(\mathcal{E}(2))=5$ from corollary 2.2 and the Riemann-Roch theorem a contradiction. By $[\mathrm{SzW} 1]$ we get sheaves with $c_{3}=0$.

5.2.1. $(0,2,4) \quad$ It can be easily obtained by the same arguments as in 4.4.2.

5.2.2. $(0,3,8) \quad$ As in 4.4 .3 . 
5.2.3. $(-1,1,1)$ By a similar argument as in 2.2 we prove: $H^{i}(\mathcal{E}(-i+$ 1)) $=0$ for $i>0$. Hence we can use the Castelnuovo-Mumford theorem for $\mathcal{E}(1)$ to derive theorem in this case.

Remark. It is known that every stable reflexive sheaf with Chern classes $(-1,1,1)$ derives from sequence $0 \rightarrow \mathcal{O}(-2) \rightarrow \mathcal{O}^{3} \rightarrow \mathcal{E}(1) \rightarrow 0$ (see [SRS1, lemma 9.4]).

5.2.4. $(-1,5,15) \quad$ Then $H^{0}(\mathcal{E}(2))=\chi(\mathcal{E}(2))=4$. Let us consider a sequence:

$$
0 \rightarrow \operatorname{ker}(e v) \longrightarrow \mathcal{O}^{4} \stackrel{e v}{\longrightarrow} \mathcal{E}(2) \longrightarrow \operatorname{coker}(e v) \rightarrow 0
$$

A sheaf $\operatorname{ker}(e v)$ is reflexive (see [OSS, II, lemma 1.1.16]) and coker(ev) is concentrated in the singular points of $\mathcal{E}(2)$, for $\left.\mathcal{E}(2)\right|_{\mathbf{P}^{2}}$ is globally generated (a weak Fano sheaf on $\mathbf{P}^{2}$ ). On the other hand theorem 3.2 yields $\left.\operatorname{ker}(e v)\right|_{\mathbf{P}^{2}} \simeq \mathcal{O}(-1) \oplus \mathcal{O}(-2)$. Therefore coker $(e v)=0$ (see [SRS1, lemma $2.7])$.

5.2.5. $(-1,7,27)$ It is easy to see that $\operatorname{dim} Y=2$ (see [A, p. 356]). There exists an ample divisor $A$ on $Y$ such that $\xi+2 H=\phi^{*} A$. We know that $A^{2} \leq(\xi+2 H)^{2} H^{2}=3, Y$ is smooth by [AW, theorem 1.4.1] and $\rho(Y)=1$, hence $A^{2}=1$. Moreover it is immediate that $\Delta(Y, A)=\operatorname{dim} Y+$ $A^{2}-h^{0}(A)=0$, so $(Y, A) \simeq\left(\mathbf{P}^{2}, \mathcal{O}_{\mathbf{P}^{2}}(1)\right)$ by $[\mathrm{Fu}]$. Therefore $\mathcal{E}(2)$ is globally generated (cf. [BW, p. 18]). This leads to the sequence 7 .

\section{$\S 6$. Small contractions}

We use notation from the last section; besides let $E$ denotes exceptional set. In this case [W1, lemma 1.1] implies $c_{1}(\mathcal{E})=-1$. By [Ka, theorem 1.1] we know that $E$ is the disjoint sum of $E_{i} \simeq \mathbf{P}^{2}, N_{E_{i} / X} \simeq \mathcal{O}_{\mathbf{P}^{2}}(-1)^{2}$ $\left(N_{E_{i} / X}\right.$-a normal bundle $E_{i}$ in $\left.X\right)$.

LEMma 6.1. In the situation as above:

$$
E \simeq \mathbf{P}^{2}, \quad N_{E / X} \simeq \mathcal{O}_{\mathbf{P}^{2}}(-1)^{2} .
$$

Proof. Considering the above remarks it is sufficient to show that $E$ is irreducible. On the contrary let us suppose that $E_{1}, E_{2}$-two disjoint 
components of $E$. Let $C=p\left(E_{1}\right) \cap p\left(E_{2}\right), \bar{C}$-the normalization of $C$. Then we have a diagram:

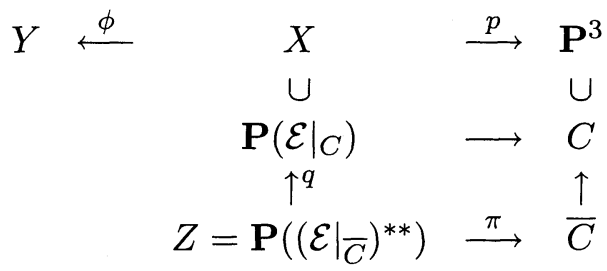

Let $C_{1}=q^{-1}\left(E_{1}\right), C_{2}=q^{-1}\left(E_{2}\right)$ be curves in $Z$ contracted by $\psi=\phi q$ to 2 different points and $\operatorname{dim} \psi(Z)=2$. Then we have:

$$
C_{1}^{2}<0, \quad C_{2}^{2}<0, \quad C_{1} \cdot C_{2} \geq 0
$$

There exist $a, b>0$ such that $a C_{1}-b C_{2}$ would be equivalent with a multiple of fiber $\pi$, so $\left(a C_{1}-b C_{2}\right)^{2}=0$, a contradiction.

THEOREM 6.2. There is only one rank 2 Fano sheaf $\mathcal{E}$ on $\mathbf{P}^{3}$ with a small contraction. It has Chern classes $c_{1}(\mathcal{E})=-1, c_{2}(\mathcal{E})=2, c_{3}(\mathcal{E})=4$ and $\mathcal{E}$ is of the form:

$$
0 \longrightarrow \mathcal{O}(-1) \longrightarrow \mathcal{O}(1)^{2} \oplus \mathcal{O} \longrightarrow \mathcal{E}(2) \longrightarrow 0 .
$$

Moreover $D_{R}=\xi+2 H$ and $E=(\xi+H)^{2}$ is the intersection of two divisors from $H^{0}(\xi+H)=H^{0}(\mathcal{E}(1))$.

Proof. First notice that $c_{2}(\mathcal{E})>0$ (cf. the remark in 3.2.1).

6.2.1. Now we will compute in two ways Chern classes of the bundle $\left.T \mathbf{P}(\mathcal{F})\right|_{E}$, where $\mathcal{F}$ is locally free and such that:

$$
0 \longrightarrow \mathcal{O}(k) \longrightarrow \mathcal{F} \longrightarrow \mathcal{E}^{\prime} \longrightarrow 0
$$

and $\mathcal{E}^{\prime}=\mathcal{E}(2), k \in \mathbf{Z}$, see fact 1.2.4. Let us assume that:

$$
\left.\mathcal{O}_{\mathbf{P}(\mathcal{F})}\left(\xi_{\mathcal{F}}\right)\right|_{E}=\mathcal{O}_{\mathbf{P}^{2}}(a)
$$

and

$$
\left.\mathcal{O}_{\mathbf{P}(\mathcal{F})}(H)\right|_{E}=\mathcal{O}_{\mathbf{P}^{2}}(d)
$$

I method: 
There are following exact sequences on $E$ :

$$
\left.0 \rightarrow N_{E / \mathbf{P}(\mathcal{E})} \longrightarrow N_{E / \mathbf{P}(\mathcal{F})} \longrightarrow N_{\mathbf{P}(\mathcal{E}) / \mathbf{P}(\mathcal{F})}\right|_{E} \rightarrow 0
$$

and

$$
\left.0 \rightarrow T \mathbf{P}^{2} \longrightarrow T \mathbf{P}(\mathcal{F})\right|_{E} \longrightarrow N_{E / \mathbf{P}(\mathcal{F})} \rightarrow 0
$$

Since $\mathbf{P}(\mathcal{E})$ is a smooth divisor from linear system $\left|\mathcal{O}_{\mathbf{P}(\mathcal{F})}\left(\xi_{\mathcal{F}}-k H\right)\right|$, so

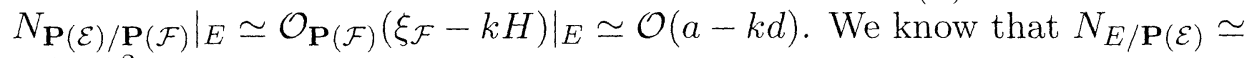
$\mathcal{O}(-1)^{2}$, hence:

$$
\begin{aligned}
c\left(\left.T \mathbf{P}(\mathcal{F})\right|_{E}\right) & =c\left(T \mathbf{P}^{2}\right) c\left(N_{E / \mathbf{P}(\mathcal{E})}\right) c\left(N_{\left.\mathbf{P}(\mathcal{E}) /\left.\mathbf{P}(\mathcal{F})\right|_{E}\right)}\right. \\
& =\left(1+3 h+3 h^{2}\right)(1-h)^{2}(1+(a-k d) h) \\
& =1+(1+a-k d) h+(a-k d-2) h^{2}
\end{aligned}
$$

II method:

We have the relative Euler sequence for $\mathcal{F}$, which we restrict to $E$ :

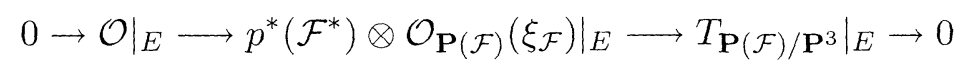

and the sequence:

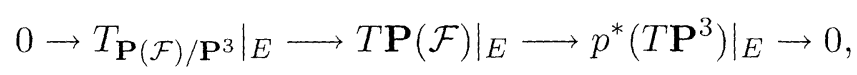

where $p: \mathbf{P}(\mathcal{F}) \rightarrow \mathbf{P}^{3}$ is a natural projection.

Remark. The same relative Euler sequence exists for a sheaf $\mathcal{E}$ on $\mathbf{P}(\mathcal{E})$.

From these sequences it is easy to compute that:

$$
\begin{aligned}
c\left(\left.T \mathbf{P}(\mathcal{F})\right|_{E}\right)= & c\left(\left.p^{*}\left(\mathcal{F}^{*}\right) \otimes \mathcal{O}_{\mathbf{P}(\mathcal{F})}\left(\xi_{\mathcal{F}}\right)\right|_{E}\right) \cdot c\left(\left.p^{*}\left(T \mathbf{P}^{3}\right)\right|_{E}\right) \\
=1+ & \left(d\left(4-c_{1}-k\right)+3 a\right) h+\left(3 a^{2}-2\left(c_{1}+k\right) a d\right. \\
& \left.\quad+\left(c_{2}+c_{1} k\right) d^{2}+4 d\left(3 a-d\left(c_{1}+k\right)\right)+6 d^{2}\right) h^{2}
\end{aligned}
$$

If we compare obtained Chern classes we get:

$$
d\left(4-c_{1}\right)+2 a=1
$$

and

$$
3 a^{2}-2\left(c_{1}-6\right) a d+\left(c_{2}-4 c_{1}+6\right) d^{2}+2-a=0
$$


If we take into account that $c_{1}=3$ we obtain:

$$
d=1-2 a
$$

and

$$
\left(4 c_{2}-33\right) a^{2}+\left(29-4 c_{2}\right) a+c_{2}-4=0 .
$$

For $\Delta=313-36 c_{2}$ is a square of integer we reduce the problem to two cases:

a) $c_{2}(\mathcal{E})=2, a=0, d=1$

b) $c_{2}(\mathcal{E})=6, a=-4, d=9$.

6.2.2. Since $\left.\mathcal{O}_{\mathbf{P}(\mathcal{E})}(H)\right|_{E}=\mathcal{O}_{\mathbf{P}^{2}}(d)$ and the length of ray $R$ equals to 1 , as a good supporting divisor $D_{R}$ one can take $2 \xi+\left(5-\frac{1}{d}\right) H$. By lemma $2.5 E \in A^{2}(\mathbf{P}(\mathcal{E}))$ can be represented as:

$$
E=b \xi H+c H^{2}+a_{1} F_{1}+\ldots+a_{k} F_{k},
$$

where $k=c_{3}$ and $a_{i}=E \cdot F_{i} \geq 0$. Next, we have the following equalities:

$$
\begin{gathered}
E \cdot D_{R}=0, \\
E \cdot\left(-K_{X}\right)^{2}=1,
\end{gathered}
$$

because of adjunction formula: $-\left.K_{X}\right|_{E}=\left(-K_{E}\right) \otimes \bigwedge^{2} N_{E / X} \simeq \mathcal{O}(1)$.

$$
E \cdot E=c_{2}\left(N_{E / X}\right)=1
$$

Hence one can easily obtain:

$$
\begin{gathered}
b=d^{2} \\
c=\frac{1}{2}\left(d-3 d^{2}\right) \\
a_{1}+\ldots+a_{k}=\frac{1}{4}\left(\left(4 c_{2}+15\right) d^{2}-8 d+1\right) \\
a_{1}^{2}+\ldots+a_{k}^{2}=4 d^{4}-d^{3}+1
\end{gathered}
$$

6.2.2.3. $\left(c_{2}(\mathcal{E})=2\right)$ Then $\mathcal{E}$ is stable, so by [SRS1, theorem 8.2] $c_{3} \leq c_{2}{ }^{2}=4$. From $(6.2 .2 .1)$ and $(6.2 .2 .2)$ we obtain $c_{3}=4, a_{1}=\ldots=$ 
$a_{4}=1$. In this case $E=(\xi+H)^{2}$. Applying corollary 2.2, theorem 3.2 and lemma 2.4.1 we get desired sequence:

$$
0 \longrightarrow \mathcal{O}(-1) \longrightarrow \mathcal{O}(1)^{2} \oplus \mathcal{O} \longrightarrow \mathcal{E}(2) \longrightarrow 0
$$

6.2.2.4. $\left(c_{2}(\mathcal{E})=6\right)$ Then:

$$
\begin{gathered}
a_{1}+\ldots+a_{k}=772 \\
a_{1}^{2}+\ldots+a_{k}^{2}=25516
\end{gathered}
$$

We now apply the inequality between arithmetic and quadratic means:

$$
23<\frac{\left(a_{1}+\ldots+a_{k}\right)^{2}}{a_{1}^{2}+\ldots+a_{k}{ }^{2}} \leq k=c_{3}
$$

Hence $c_{3} \geq 24$. It can be easily proved, by the Nakai-Moishezon criterion on $P\left(\left.\mathcal{E}\right|_{\mathbf{P}^{2}}\right)$, that $H^{0}\left(\left.\mathcal{E}(1)\right|_{\mathbf{P}^{2}}\right)=0$ and $H^{0}(\mathcal{E}(1))=0$.

By [SRS1, theorem 6.2] we get $H^{1}\left(\left.\mathcal{E}(2)\right|_{\mathbf{P}^{2}}\right)=0$; also we know that $H^{2}\left(\left.\mathcal{E}(2)\right|_{\mathbf{P}^{2}}\right)=0$, hence $H^{0}\left(\left.\mathcal{E}(2)\right|_{\mathbf{P}^{2}}\right)=\chi\left(\left.\mathcal{E}(2)\right|_{\mathbf{P}^{2}}\right)=3$. On the other hand [SRS3, theorem 1.1] yields $H^{2}(\mathcal{E}(2)) \leq 1$ and by Riemann-Roch theorem:

$$
5 \leq \chi(\mathcal{E}(2))=\frac{1}{2}\left(28+c_{3}-42\right) \leq h^{0}(\mathcal{E}(2))+1
$$

and

$$
0=H^{0}(\mathcal{E}(1)) \longrightarrow H^{0}(\mathcal{E}(2)) \longrightarrow H^{0}\left(\left.\mathcal{E}(2)\right|_{\mathbf{P}^{2}}\right),
$$

we get $h^{0}(\mathcal{E}(2)) \leq h^{0}\left(\left.\mathcal{E}(2)\right|_{\mathbf{P}^{2}}\right)=3$, a contradiction. Therefore this case cannot occur.

\section{$\S 7$. Fano sheaves on 3-dimensional quadric}

Now we will be occupied with the case of Fano sheaves over the 3dimensional quadric. The following result may be proved in much the same way as theorem 4.2 and lemmas $4.2,5.1,6.1$ and theorem 6.2:

LEMmA 7.1. Let $\mathcal{E}$ be a Fano sheaf of rank 2 on $\mathbf{Q}_{3}$ with $c_{1}=0$ or -1 . If the other contraction of $\mathbf{P}(\mathcal{E})$ is fiber then either $\mathcal{E}=\mathcal{O}^{2}$ or $\mathcal{E}=\mathbf{S}$ (the spinor bundle) or $\mathcal{E}(1)$ is nef and $\mathcal{E}$ has Chern classes $(0,2,0)$, $(0,4,8),(0,6,16)$ or $(-1,2,2)$. If this contraction is divisorial then either $\mathcal{E}=\mathcal{O} \oplus \mathcal{O}(-1), \mathcal{O}(-1) \oplus \mathcal{O}(1)$ or $\mathcal{E}(1)$ is nef and $\mathcal{E}$ has Chern classes $(0,2,4),(0,1,1),(0,2,2)$ or $(0,3,5)$. The small contraction does not occur. 
Theorem 7.2. Classification of rank 2 Fano sheaves on $\mathbf{Q}_{3}$ :

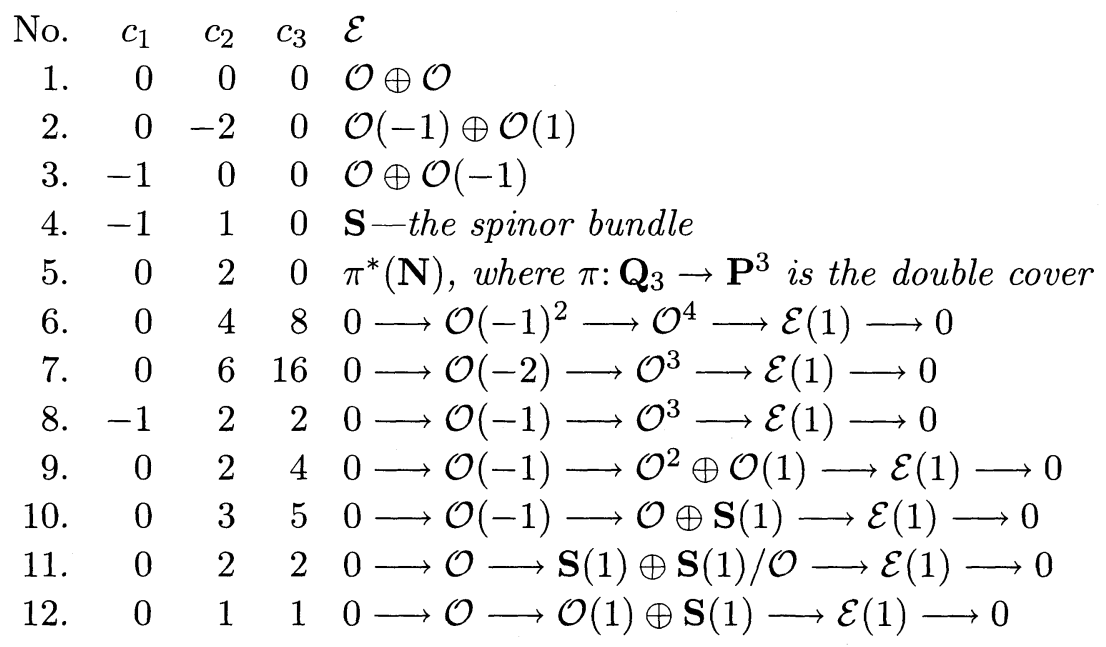

Proof. To prove this theorem we will extensively use [AO, theorem 6.7]. We will consider some cases according to the Chern classes of the sheaf $\mathcal{E}$.

7.2.1. In cases $(0,2,0),(0,4,8),(0,6,16)$ and $(-1,2,2)$ it can be easily proved by lemma 8.1 and considerations similar to those in 3.2 .0 , that $\mathcal{E}(1)$ is globally generated. In case $(0,2,0)$ we can now use [SzW1, proposition 3.2]. From the remaining cases we will prove the theorem only for the most difficult case: $(0,4,8)$.

First let us remark that $h^{0}(\mathcal{E}(1))=4$ and $h^{i}(\mathcal{E}(1))=0$ for $i>0$. One can also prove, using divisorial sequence, that $h^{1}(\mathcal{E}(-1))=0$. So there exists a bundle $\mathcal{F}$ such that we have the following sequence:

$$
0 \longrightarrow \mathcal{F} \longrightarrow \mathcal{O}^{4} \longrightarrow \mathcal{E}(1) \longrightarrow 0 .
$$

So it follows that $h^{i}(\mathcal{F}(-i))=0$ for $i=0,1,2$. Now using [AO, theorem 6.7] we easily prove that $\mathcal{F}=\mathcal{O}(-1)^{2}$,

7.2.2. $(0,1,1) \quad$ From the Kawamata-Viehweg vanishing theorem $h^{i}(\mathcal{E})$ - 0 for $i>0$ and so $h^{0}(\mathcal{E})=1$. The section of $\mathcal{E}$ vanishes along a line $L$ and we have the following exact sequence:

$$
0 \longrightarrow \mathcal{O} \longrightarrow \mathcal{E} \longrightarrow J_{L} \longrightarrow 0 .
$$

From this sequence $h^{2}\left((\mathcal{E}(1))^{*}\right)=0$, so $\mathcal{E}(1)$ extends to a sheaf $\mathcal{F}$. One can easily prove that $h^{i}(\mathcal{F}(-i-1))=0$ for $i=0,1,2$, so from [AO, theorem 6.7] it follows that $\mathcal{F}=\mathbf{S}(1) \oplus \mathcal{O}(1)$. 
7.2.3. $(0,2,4)$ Quite similarly as above we can show the existence of the sequence:

$$
0 \longrightarrow \mathcal{O} \longrightarrow \mathcal{E} \longrightarrow J_{C} \longrightarrow 0
$$

where $C$ is a smooth conic. From this $\operatorname{dim} \operatorname{Ext}^{1}(\mathcal{E}(2), \mathcal{O})=h^{2}(\mathcal{E}(-1))=1$ and therefore there exists a non-trivial extension $\mathcal{F}$ :

$$
0 \longrightarrow \mathcal{O} \longrightarrow \mathcal{F} \longrightarrow \mathcal{E}(2) \longrightarrow 0
$$

Because $h^{0}(\mathcal{F}(-2))=1$ we have the sequence:

$$
0 \longrightarrow \mathcal{O} \longrightarrow \mathcal{F}(-2) \longrightarrow \mathcal{G} \longrightarrow 0
$$

for some sheaf $\mathcal{G}$. Now because $h^{i}(\mathcal{G}(-i))=0$ for $i=0,1,2$, we have $\mathcal{G}=\mathcal{O}(-1)^{2}$.

7.2.4. $(0,2,2) \quad$ The spectrum of $\mathcal{E}$ is $\{-1,-1\}$, so $h^{2}\left((\mathcal{E}(1))^{*}\right)=0$ (see $[\mathrm{ES}])$ and $\mathcal{E}(1)$ extends to $\mathcal{F}$, such that $h^{i}(\mathcal{F}(-i-1))=0$ for $i=0,1$, but $h^{2}(\mathcal{F}(-3))=1$. Hence there exists a non-trivial extension $\mathcal{G} \in \operatorname{Ext}^{1}(\mathcal{F}, \mathcal{O})$. Now $h^{i}(\mathcal{G}(-i-1))=0$ for $i=0,1,2$ and from [AO, theorem 6.7] it follows that $\mathcal{G}=\mathbf{S}(1) \oplus \mathbf{S}(1)$.

7.2.5. $(0,3,5) \quad$ Similar to 7.2 .4 .

This finishes a proof of theorem 7.2 .

\section{$\S 8$. Fano sheaves on other Fano 3-folds}

This is quite similar to the considerations in the last section.

Lemma 8.1. Let $\mathcal{E}$ be a Fano sheaf of rank 2 on $V_{d}$ with $c_{1}=0$ or 1 . Then $\mathcal{E}$ is nef. Moreover if the other contraction of $\mathbf{P}(\mathcal{E})$ is fiber then $\mathcal{E}$ has Chern classes $(0,0,0),(1, d, d)$ or $d=4$ and $(1,2,0)$, or $d=5$ and $(1,3,1)$. If this contraction is divisorial then the Chern classes are as follows: $(1, c, c)$, where $c \geq 0$, or $d=5$ and $(1,2,0)$. Small contractions do not occur.

Proof. Proof is similar to that of lemma 8.1, but if $c_{1}=0$ one can simplify computations using [BW, lemma 4.6]. Because $\mathcal{E}(1)$ is ample then from this lemma for any line $L$ :

$$
2=L \cdot \operatorname{det} \mathcal{E}(1) \geq \operatorname{rank} \mathcal{E}+(\text { number of singular points of } \mathcal{E} \text { on } L)
$$


Because $V_{d}$ is covered by lines it follows that $\mathcal{E}$ is locally free. First we show that $\mathcal{E}$ is nef. Then it is easy to see that $c_{2} \geq 0$, for from the Hodge index theorem (see [Fu, theorem 0.4.6]) we have:

$$
\left(d-c_{2}\right) d=\xi^{2}(\xi H) \cdot H^{2}(\xi H) \leq\left(\xi^{2} H^{2}\right)^{2}=d^{2} .
$$

Then one can easily compute Chern classes of $\mathcal{E}$.

THEOREM 8.2. Classification of rank 2 Fano sheaves on $V_{d}$ :

$$
\begin{aligned}
& \text { No. } \quad c_{1} \quad c_{2} \quad c_{3} \quad \mathcal{E} \\
& \text { 1. } 0 \quad 0 \quad 0 \quad 0 \quad \mathcal{O} \oplus \mathcal{O} \\
& \text { 2. } \quad 0 \quad 1 \quad 0 \quad \mathcal{O} \oplus \mathcal{O}(1) \\
& \text { 3. } 1 \quad d \quad d \quad 0 \longrightarrow \mathcal{O}(-1) \longrightarrow \mathcal{O}^{3} \longrightarrow \mathcal{E} \longrightarrow 0 \\
& \text { For } d=4 \text { we have also the following bundles: } \\
& \text { 4. } \quad 1 \quad 2 \quad 0 \quad \mathcal{E} \text {-stable bundles (see [SzW4]) } \\
& \text { For } d=5 \text { we have also the following sheaves: } \\
& \text { 5. } 122 \quad 0 \quad \mathcal{F} \text {-the only stable bundle with these Chern classes } \\
& \text { (see }[\mathrm{SzW} 4] \text { ) } \\
& \text { 6. } 1310 \longrightarrow \mathcal{F}(-1) \longrightarrow \mathcal{O}^{4} \longrightarrow \mathcal{E} \longrightarrow 0 \text {, } \\
& \text { where } \mathcal{F} \text { is the bundle from } 5 \text {. }
\end{aligned}
$$

Proof. First, we show that for $\mathcal{E}$ with Chern classes $(1, c, c)$ we have $\mathcal{E}=\mathcal{O} \oplus \mathcal{O}(1)$. Let us remark that by Kawamata-Viehweg theorem and Riemann-Roch formula we have $h^{0}(\mathcal{E}(-1))=1$ and $h^{2}\left(\mathcal{E}^{*}\right)=0$. It follows that $\mathcal{E}$ extends to a locally free sheaf $\mathcal{F}$ such that $\mathcal{F}$ is nef, $c_{1}(\mathcal{F})=1$ and $h^{0}(\mathcal{F}(-1))=1$. If the section of $\mathcal{F}(-1)$ vanishes in some points then we can take a conic $C$, which meets the zero locus of this section in a finite number of points. Then:

$$
\left.\mathcal{F}(-1)\right|_{C}=\mathcal{O}(a) \oplus \mathcal{O}(b) \oplus \mathcal{O}(c)
$$

where $a+b+c=-4, a>0$ and $b, c \geq-2$ (because $\mathcal{F}$ is nef). So we get a contradiction and this section vanishes nowhere. Hence we get an exact sequence:

$$
0 \longrightarrow \mathcal{O} \longrightarrow \mathcal{F}(-1) \longrightarrow \mathcal{G} \longrightarrow 0
$$

where $\mathcal{G}$ is locally free. One can check that $\mathcal{G}(1)$ is nef and $c_{1}(\mathcal{G})=0$, so $\mathcal{G}=\mathcal{O}(-1)^{2}$ and therefore $\mathcal{E}=\mathcal{O} \oplus \mathcal{O}(1)$. In all remaining cases one can show that $\mathcal{E}$ is globally generated and computing $h^{0}(\mathcal{E})$ one easily shows the theorem. 
Let us also remark the following fact about uniform vector bundles with $c_{1}=0$ on $V_{d}$ (cf. [OSS, theorem 3.2.1]):

Proposition 8.3. Let $\mathcal{E}$ be a vector bundle on $V_{d}$ such that $c_{1}=0$ and for any line $L:\left.\mathcal{E}\right|_{L}=\mathcal{O} \oplus \cdots \oplus \mathcal{O}$. Then $c_{2}(E)=0$. Moreover if $\mathcal{E}$ has rank 2 , then it is trivial.

Proof. Let $T$ be a scheme parametrizing a family of lines on $V_{d}$ and let $S$ be an incidence variety of lines on $V_{d}$. T is known to be a smooth surface. We have natural projections $p: S \rightarrow V_{d}$ and $q: S \rightarrow T$. Let $L$ be a general line on $V_{d}$ and $C=q\left(p^{-1}(L)\right)$ be a curve, parametrizing lines meeting line $L$. Now let us take a normalization $\bar{C}_{0}$ of some irreducible 1-dimensional component $C_{0}$ of $C$ and the pull back $\bar{F} \stackrel{q^{\prime}}{\rightarrow} \bar{C}_{0}$ of universal $\mathbf{P}^{1}$-bundle $q^{-1}(C) \stackrel{q}{\rightarrow} C$. Then we have the following diagram:

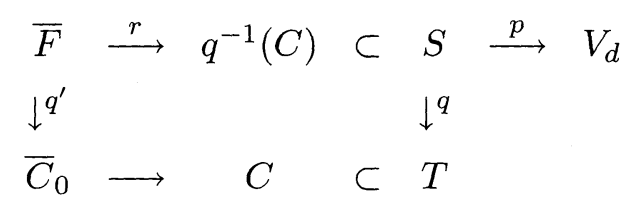

Let us remark that $q^{\prime}$ is a $\mathbf{P}^{1}$-bundle, so $\bar{F}$ is a smooth surface. It is also easy to see that $q^{\prime}$ has a section $s$, which sends a point of $\bar{C}_{0}$ to a point of intersection of corresponding line with $L$ (this is well defined over open subset of curve $\bar{C}_{0}$ and so can be extended to a section on the whole curve). Let us denote $p^{\prime}=r p: \bar{F} \rightarrow V_{d}$ and consider bundle $\mathcal{F}=p^{\prime *}(\mathcal{E})$. This bundle is trivial over fibers of $q^{\prime}$, so there exists a bundle $\mathcal{G}$ on $\bar{C}_{0}$ such that $\mathcal{F}=q^{\prime *}(\mathcal{G})$. We claim that now $\mathcal{G}$ is trivial. Indeed, we have (compare with [OSS, theorem 3.2.1]):

$$
\mathcal{O} \bar{C}_{0}^{r}=\left(p^{\prime} s\right)^{*}(\mathcal{E})=s^{*}\left(q^{\prime *}(\mathcal{G})\right)=\left(q^{\prime} s\right)^{*}(\mathcal{G})=\mathcal{G}
$$

It follows that $\mathcal{F}$ is trivial and from the projection formula (because $p^{\prime}(\bar{F})$ is a generator of $\left.\operatorname{Pic}\left(V_{d}\right)\right)$ we get $c_{2}(\mathcal{E})=0$.

If the rank of sheaf $\mathcal{E}$ is two then one can easily see that if $\mathcal{E}$ is nontrivial then $h^{0}(\mathcal{E})=h^{0}\left(\mathcal{E}^{*}\right)=0$. Indeed, otherwise the section of $\mathcal{E}$ would be non-vanishing in all points (because of the splitting type on lines) and we would get trivial subbundle of $\mathcal{E}$. Now from the Bogomolov inequality for rank 2 stable bundles on $V_{d}$ (see [L]) we have $c_{2}>0$, a contradiction. So the theorem is proved. 
Now we will study Fano sheaves on Fano threefolds of the first kind (i.e., such that $-K_{X}=H$ and $\operatorname{Pic} X=\mathbf{Z}[H]$ ).

THEOREM 8.4. If $\mathcal{E}$ is a Fano sheaf of rank 2 on $X$, then it is just $\mathcal{O}^{2}$.

Proof. First let us remark that if $c_{1}=-1$ then by [BW, lemma 4.6] the number of singular points on any conic is equal to 0 . Because $X$ is covered by conics it follows that $\mathcal{E}$ is locally free. Then as before we show that $\mathcal{E}$ is nef. Now by the same argument as in lemma 8.1 one can show that if $c_{1}=0$ then $c_{2}=0$. It makes easier computations, that the Chern classes of $\mathcal{E}$ must be $(0,0,0)$ and the other contraction of $\mathbf{P}(\mathcal{E})$ is fiber. Now it is straightforward that $\mathcal{E}=\mathcal{O}^{2}$.

\section{$\S 9$. The geometry of $\mathbf{P}(\mathcal{E})$}

In this section we study geometry of $X=\mathbf{P}(\mathcal{E})$, where $\mathcal{E}$ always denotes a rank 2 Fano sheaf. Firstly, let us remark that for locally free sheaves $\mathcal{F}$ and $\mathcal{G}$ a manifold $\mathbf{P}(\mathcal{F} \oplus \mathcal{G})$ is a joint of manifolds $\mathbf{P}(\mathcal{F})$ and $\mathbf{P}(\mathcal{G})$. In our situation we usually obtain a sequence of the form:

$$
0 \longrightarrow \mathcal{G} \longrightarrow \mathcal{F} \longrightarrow \mathcal{E} \longrightarrow 0
$$

where $\mathcal{F}$ is a sum of some bundles, whose projectivisations we know. Hence $\mathbf{P}(\mathcal{E})$ can be embedded into $\mathbf{P}(\mathcal{F})$ "by means of $\mathcal{G}$ " and $\mathbf{P}(\mathcal{F})$ is easy to describe. Moreover we already know the good supporting divisor $D_{R}$ and we know that it is globally generated. Hence it defines a morphism, which in all our cases occurs to be the contraction $\phi$ (usually even if $D_{R}$ is generated by global sections one has to take its Stein factorization to obtain a contraction of extremal ray). These remarks allow us to give a completes description of $\mathbf{P}(\mathcal{E})$ and the contraction $\phi$.

For the convenience of the reader we give here a complete description of projectivizations of Fano sheaves over $\mathbf{P}^{3}$ and more interesting examples among others.

\subsection{Complete description of $\mathbf{P}(\mathcal{E})$ for sheaves on $\mathbf{P}^{3}$.}

We use here notation from theorems 4.4, 5.2 and 6.2.

9.1.1. (Divisorial contractions) In this case one can easily compute all important invariants of contraction and of manifolds $X$ and $Y$. Basic informations are gathered in the following table (numbers in the table are the same as that from theorem 4.4): 


\begin{tabular}{|c|c|c|c|c|c|c|c|}
\hline No. & $D_{R}$ & $E$ & $A^{4}$ & $\Delta(Y, A)$ & $\operatorname{dim} \phi(E)$ & $\operatorname{deg} \phi(E)$ & $h^{0}\left(D_{R}\right)$ \\
\hline 1 & $\xi+H$ & $\xi$ & 1 & 0 & 0 & 1 & 5 \\
2 & $\xi+H$ & $\xi-H$ & 8 & 1 & 0 & 1 & 11 \\
3 & $\xi+2 H$ & $\xi-H$ & 27 & 7 & 0 & 1 & 21 \\
4 & $\xi+H$ & $\xi$ & 2 & 0 & 1 & 1 & 6 \\
5 & $\xi+2 H$ & $\xi+H$ & 3 & 1 & 1 & 1 & 6 \\
6 & $\xi+2 H$ & $\xi$ & 12 & 4 & 1 & 1 & 12 \\
7 & $\xi+2 H$ & $\xi+H$ & 5 & 1 & 2 & 2 & 8 \\
8 & $\xi+2 H$ & $2 \xi+3 H$ & 2 & 0 & 2 & 5 & 6 \\
9 & $\xi+2 H$ & $\xi+H$ & 4 & 1 & 2 & 1 & 7 \\
10 & $\xi+2 H$ & $3 \xi+5 H$ & 1 & 0 & 2 & 6 & 5 \\
\hline
\end{tabular}

Because in all cases $\Delta(Y, A)=g(Y, A)$ in the table there is no separate place for $g(Y, A)$.

A description of the manifold $\mathbf{P}(\mathcal{E})$ :

1. A blow up of $\mathbf{P}^{4}$ along a point, $Y=\mathbf{P}^{4}$,

2. A blow up of the cone $Y$ in $\mathbf{P}^{10}$ over Veronese embedding $\mathbf{P}^{3} \underset{|\mathcal{O}(2)|}{\hookrightarrow} \mathbf{P}^{9}$ along its vertex (a point),

3. A blow up of the cone $Y$ in $\mathbf{P}^{20}$ over Veronese embedding $\mathbf{P}^{3} \underset{|\mathcal{O}(3)|}{\hookrightarrow} \mathbf{P}^{19}$ along its vertex (a point),

4. A blow up of $Y=\mathbf{Q}_{4}$ along a line $\mathbf{P}^{1}$,

5. As one can easily see $\mathbf{P}\left(\mathcal{O}(1) \oplus \mathcal{O}^{2}\right)$ is also a Fano manifold, which contains $\mathbf{P}(\mathcal{E})$ as a divisor of type $(2,1)$ (i.e. $\left.\mathbf{P}(\mathcal{E}) \in\left|\xi_{\left(\mathcal{O}(1) \oplus \mathcal{O}^{2}\right)}+2 H\right|\right)$. $\mathbf{P}\left(\mathcal{O}(1) \oplus \mathcal{O}^{2}\right)$ is a blow up of $\mathbf{P}^{5}$ along a line $L$. Because the trace of linear system $\left|\xi_{\left(\mathcal{O}(1) \oplus \mathcal{O}^{2}\right)}+2 H\right|_{\mathbf{P}(\mathcal{E})} \mid$ is a complete linear system $\left|D_{R}\right|$, so the restriction of a contraction $\phi^{\prime}: \mathbf{P}\left(\mathcal{O}(1) \oplus \mathcal{O}^{2}\right) \rightarrow \mathbf{P}^{5}$ to $\mathbf{P}(\mathcal{E})$ is also a contraction: $\left.\phi^{\prime}\right|_{\mathbf{P}(\mathcal{E})}=\phi$. The image $\phi(Y)$ is a hypercubic in $\mathbf{P}^{5}$ (see the table) and the exceptional divisor $E$ of $\phi$ is contracted onto a line $L$ (because it is 1-dimensional). Because $D_{R} E H^{2}=2$ it follows that all fibers of map $\left.\phi\right|_{E}$ are 2-dimensional quadrics (possibly singular).

6. $\mathbf{P}(\mathcal{E})$ is a divisor of type $(1,1)$ in $\mathbf{P}\left(\mathcal{O}(2) \oplus \mathcal{O}^{2}\right)$ (a blow up of the cone in $\mathbf{P}^{11}$ over $\mathbf{P}^{3} \underset{|\mathcal{O}(2)|}{\hookrightarrow} \mathbf{P}^{9}$ along a "vertex" $\left.L=\mathbf{P}^{1}\right)$. Similarly as above 
one can see that $Y$ is a divisor in this cone, containing line $L$, as an image of the exceptional divisor $E$.

7. A complete intersection of two divisors of types $(0,1)$ in $T=\mathbf{P}(\Omega(2) \oplus$ $\mathcal{O}(1))$. This last manifold $T$ is a Fano variety - a resolution of a rational map $q: G(1,4) \rightarrow \mathbf{P}^{3}$. If we fix a hyperplane $H$ in $\mathbf{P}^{4}$ then the map $q$ can be easily described: it sends a line $l$ to its intersection with this hyperplane. $T$ admits two contractions: one onto $\mathbf{P}^{3}$ and the other (which is a blow up along $G(1, H)=4$-dimensional quadric) onto a Grassmanian $G(1,4)$. Now it is easy to see that $E$ is contracted onto 2-dimensional quadric in $Y$ and all fibers of $\left.\phi\right|_{E}$ are just $\mathbf{P}^{1}$. It follows that $\phi$ is a blow up of a smooth linear section of Grassmanian $G(1,4)$ along a smooth 2-dimensional quadric.

8. It is convenient to look at the cases a) and b) from theorem 4.4 at the same time. In both cases $\mathbf{P}(\mathcal{E})$ is an intersection of two divisors $D_{1} \in\left|\xi_{\Omega(2) \oplus \mathcal{O}}\right|$ and $D_{2} \in\left|\xi_{\Omega(2) \oplus \mathcal{O}}+H\right|$ in $\mathbf{P}(\Omega(2) \oplus \mathcal{O}) . \mathbf{P}(\Omega(2) \oplus \mathcal{O})$ is a joint of $\mathbf{P}^{3}$ and the incidence variety of lines in $\mathbf{P}^{3}$. The linear system $\left|\xi_{\Omega(2) \oplus \mathcal{O}}\right|$ defines a contraction of $\mathbf{P}(\Omega(2) \oplus \mathcal{O})$ to a cone over 4-dimensional quadric $G(1,3)$ (the section $F$ corresponding to a trivial factor of $\Omega(2) \oplus \mathcal{O}$ is contracted to a vertex $P$, while rest of fibers are lines). The restriction of the morphism defined by this system to $X$ gives us contraction $\phi: X \rightarrow Y$.

If $D_{1}$ contains $F$, then we obtain case b). In this case $D_{2}$ cuts out a plane on $F$, which is contracted to $P$, and $Y$ is a cone over a smooth 3 -dimensional quadric. $\phi$ has one 2-dimensional fiber and the rest of non-trivial fibers are lines. One can also prove that the image of exceptional divisor $\phi(E)$ is a smooth surface of degree 5 .

If $D_{1}$ does not contain $F$, then we obtain case a). The other divisor $D_{2}$ cuts out line in $F$, so all non-trivial fibers are 1-dimensional. It follows that $\phi$ is a blow up of smooth quadric $Y$ along a smooth surface of degree 5 .

One can show that this surface is a Del Pezzo surface embedded by its anticanonical system into $\mathbf{P}^{5}$.

9. A complete intersection of two divisors of type $(1,1)$ in $\mathbf{P}\left(\mathcal{O}(1) \oplus \mathcal{O}^{3}\right)$ (a blow up of $\mathbf{P}^{6}$ along a plane $\mathbf{P}^{2}$ ). One can easily see that there are no 2-dimensional fibers and $\phi$ is a blow up of a smooth complete intersection of two hyperquadrics in $\mathbf{P}^{6}$ along the plane. 
10. $\mathbf{P}(\mathcal{E})$ is a complete intersection of three divisors of type $(1,1)$ in $\mathbf{P}^{3} \times \mathbf{P}^{4}$ and $\phi$ is a projection onto the second component $Y=\mathbf{P}^{4}$. In this case one can easily describe situation: $X$ is given by three equations:

$$
\sum_{i, j} a_{i j k} x_{i} y_{j}=0 \quad k=1,2,3,
$$

where $x_{i}$ are coordinates in $\mathbf{P}^{3}, y_{j}$ are coordinates in $\mathbf{P}^{4}$ and $a_{i j k}$ are general coefficients. Over point $\left[y_{0}, \ldots, y_{4}\right]$ there is generically one point (an intersection of three hyperplanes in $\mathbf{P}^{3}$ ), but if these equations $(*)$ are dependent, then we get $\mathbf{P}^{1}$. This last condition is described by 4 equations of degree 3 in $\mathbf{P}^{4}$ :

$$
\operatorname{det}_{j \neq l}\left(\sum_{j} a_{i j k} y_{j}\right)=0, \quad l=1, \ldots 4 .
$$

Now it is easy to see that the surface $\phi(E)$ is a blow up of $\mathbf{P}^{2}$ in 10 points and $\phi$ is a blow up of $\mathbf{P}^{4}$ along this surface.

9.1.2. (Fiber contractions) In this case all necessary informations can be read off the form of sheaf $\mathcal{E}$. Numbers coincide with that from theorem 5.2.

A description of the manifold $\mathbf{P}(\mathcal{E})$ :

1. $\mathbf{P}^{1} \times \mathbf{P}^{3} ; Y=\mathbf{P}^{1}$,

2. A divisor of type $(0,1)$ in the incidence variety $\mathbf{P}(\Omega(2)) ; Y=\mathbf{Q}_{3}$ a complete description of this contraction can be found in [SzW1, pp. 202-203],

3. An intersection of two divisors of types $(1,1)$ in $\mathbf{P}^{3} \times \mathbf{P}^{3} ; \phi: X \rightarrow Y=$ $\mathbf{P}^{3}$ is the projectivisation of a sheaf of the same form as $\mathcal{E}$,

4. A divisor of type $(2,1)$ in $\mathbf{P}^{3} \times \mathbf{P}^{2}$. Contraction $\phi: X \rightarrow Y=\mathbf{P}^{2}$ is a quadric bundle.

5. A divisor of type $(1,1)$ in $\mathbf{P}^{2} \times \mathbf{P}^{3} ; \phi: X \rightarrow Y=\mathbf{P}^{2}$ is a projectivisation of the bundle $T_{\mathbf{P}^{2}}(-1) \oplus \mathcal{O}$ on $\mathbf{P}^{2}$ (see [SzW3, p. 89]),

6. An intersection of two divisors of types $(1,1)$ and $(2,1)$ in $\mathbf{P}^{3} \times \mathbf{P}^{3}$. Contraction $\phi: X \rightarrow Y=\mathbf{P}^{3}$ is a projection onto the second factor and it is a conic bundle. 
7. A divisor of type $(3,1)$ in $\mathbf{P}^{3} \times \mathbf{P}^{2}$; all fibers of contraction $\phi: X \rightarrow$ $Y=\mathbf{P}^{2}$ are cubics in $\mathbf{P}^{3}$.

9.1.3. (Small contractions) From theorem 6.2 we have only one such sheaf that the other contraction $\phi: X \rightarrow Y$ is small. In this case $\mathbf{P}(\mathcal{E})$ is a divisor of type $(1,1)$ in $\mathbf{P}\left(\mathcal{O}(1)^{2} \oplus \mathcal{O}\right)$. This last manifold is Fano-it is a blow up of the cone in $\mathbf{P}^{8}$ over embedding $\mathbf{P}^{1} \times \mathbf{P}^{3} \hookrightarrow \mathbf{P}^{7}$ along the vertex (which is a point).

\subsection{Some other examples of scrolls}

9.2.1. Here we will study geometry of Fano sheaves over quadric $\mathbf{Q}_{3}$. This is very easy for sheaves $1-8$ from theorem 7.2 , so we omit it. For others cases we have the following table (numbers in the table correspond to these from theorem 7.2):

\begin{tabular}{|c|c|c|c|c|c|c|c|}
\hline No. & $D_{R}$ & $E$ & $A^{4}$ & $\Delta(Y, A)$ & $\operatorname{dim} \phi(E)$ & $\operatorname{deg} \phi(E)$ & $h^{0}\left(D_{R}\right)$ \\
\hline 9 & $\xi+H$ & $\xi$ & 4 & 1 & 1 & 1 & 7 \\
10 & $\xi+H$ & $3 \xi+2 H$ & 1 & 0 & 2 & 8 & 5 \\
11 & $\xi+H$ & $2 \xi+H$ & 1 & 0 & 2 & 4 & 6 \\
12 & $\xi+H$ & $\xi$ & 5 & 1 & 2 & 1 & 8 \\
\hline
\end{tabular}

A description of the manifold $X=\mathbf{P}(\mathcal{E})$ :

9. $X$ is a divisor of type $(1,1)$ in $M=\mathbf{P}\left(\mathcal{O}^{2} \oplus \mathcal{O}(1)\right)$. Manifold $M$ is Fano and has an extremal ray contraction (given by a linear system $\left.\xi_{\mathcal{O}^{2} \oplus \mathcal{O}(1)}\right)$ onto a cone with vertex $L=\mathbf{P}^{1}$ over 3-dimensional quadric $\mathrm{Q}_{3}$. A restriction of this contraction to $X$ is again a contraction. $X$ is contracted onto the intersection of two quadrics in $\mathbf{P}^{6}$ and the exceptional divisor is contracted onto the line $L$. All non-trivial fibers of $\phi$ are quadrics (because $D_{R} E H^{2}=2$ ).

10. $X$ is a divisor of type $(1,1)$ in $\mathbf{P}(\mathcal{O} \oplus \mathbf{S}(1))$ (i.e. $X \in \mid \xi_{\mathcal{O} \oplus \mathbf{S}(1)}+$ $H \mid)$. This last variety has a contraction given by the linear system $\left|\xi_{\mathcal{O} \oplus \mathbf{S}(1)}\right|$. This contraction has one 3-dimensional fiber corresponding to the trivial factor of $\mathcal{O} \oplus \mathbf{S}(1)$ whereas all other fibers are lines. The restriction of this contraction to $X$ gives us contraction $\phi$ of $X$. It is a divisorial contraction with one 2-dimensional fiber (all other nontrivial fibers are lines). This 2-dimensional fiber is a quadric, which is either smooth or singular (a cone over plane conic). Moreover both this cases occur as can be seen by considering a degeneracy locus. In 
this case the exceptional divisor is contracted onto a singular surface (non-normal) of degree 8.

First we will prove that in cases 11 and 12 there are no 2-dimensional fibers. Let's on the contrary assume that there is such fiber $F$. In both these cases we have exact sequence of the form:

$$
0 \longrightarrow \mathcal{O} \longrightarrow \mathcal{F} \longrightarrow \mathcal{E} \longrightarrow 0
$$

for some bundle $\mathcal{F}$. Then we know all the possibilities for $F$ (see [AW2]). Equality $H \cdot C=1$ for curves contracted by $\phi$ then implies $\left.H\right|_{F}=\mathcal{O}_{F}(1)$ and so $\left.p\right|_{F}: F \rightarrow p(F)$ is an isomorphism. Because there are no $\mathbf{P}^{2}$ 's in $\mathbf{Q}_{3}$ we conclude that $F$ is a quadric (possibly singular). Moreover there exists a section of $\left.p\right|_{F}$, which corresponds to a bundle epimorphism:

$$
\left.\mathcal{F}\right|_{p(F)} \rightarrow \mathcal{O}_{p(F)} \rightarrow 0
$$

But $\left.\mathcal{F}^{*}\right|_{p(F)}$ has no sections so our assuption was false.

We proved that there are no 2-dimensional fibers, so $\phi$ is a blow up of a smooth 4-fold along a smooth surface. Now we will study cases 11 and 12 separately.

11. $X$ is a blow up of a smooth 4-dimensional quadric along a surface of degree 4. One can also prove that this surface is an intersection of two quadrics naturally embedded in $\mathbf{Q}_{4}$.

12. One can easily see that $X$ is a blow up of a smooth linear section of Grassmanian $G(1,4) \hookrightarrow \mathbf{P}^{9}$ along a plane $\mathbf{P}^{2}$ (see the table). It is also a smooth divisor from the linear system $\left|\xi_{\mathcal{O}(1) \oplus \mathbf{S}(1)}\right|$ in $\mathbf{P}(\mathcal{O}(1) \oplus \mathbf{S}(1))$.

9.2.2. Among all other scrolls the only non-trivial case is case 5 from theorem 8.2. The geometry of this last scroll is fairly complicated and partially described in [SzW4]. In this case $\mathbf{P}(\mathcal{F})$ is a blow up of $\mathbf{P}^{4}$ along a Veronese surface. One can also find the normal bundle of this surface in $\mathbf{P}^{4}$ (using classification of weak Fano bundles on $\mathbf{P}^{2}$; see theorem 3.2). It is $\left(\mathcal{O}(1) \oplus \mathcal{O}^{2}\right) / \mathcal{O}(-2)$. Projectivisation of this bundle is the exceptional divisor, which occurs to be the incidence variety of lines in $V_{d}$.

\section{Appendix: Fano bundles of rank 2 on $\mathbf{P}^{2}$ and on Fano threefolds.}

A.1. Let $\mathcal{E}$ be a Fano bundle of rank 2 on $\mathbf{P}^{2}$ and let $\phi: \mathbf{P}(\mathcal{E}) \rightarrow Y$ be the "other" contraction of an extremal ray. Set $D_{R}=\xi+u H=\phi^{*} A, u \in \mathbf{Q}-\mathbf{a}$ 
good supporting divisor and assume that $c_{1}(\mathcal{E})=0,0<c_{2}(\mathcal{E}) \leq 6$-we can do that because otherwise we use [SzW2, fact 2.3]. It can be easily proved that $u \geq 0$-compare with lemma 2.7. Write the table of intersections on $\mathbf{P}(\mathcal{E})$ :

$$
H^{3}=0, \quad H^{2} \xi=1, \quad H \xi^{2}=0, \quad \xi^{3}=-c_{2}
$$

Then:

$$
D_{R}^{3}=3 u^{2}-c_{2} \text {. }
$$

We know that $\phi$ is either fiber or divisorial (small contractions do not occur for smooth 3 -folds). We will consider these two cases separately:

a) $\phi$ is of fiber type. Then $D_{R}^{3}=0,0<c_{2}=3 u^{2} \leq 6$, hence $u=1$ and $c_{2}=3$. Since $D_{R}^{2} H=2$ we have $\operatorname{dim} Y=2$ and $Y$ is a smooth surface, by classification of contractions from smooth 3-folds. But $\rho(Y)=1$ and $A^{2} \leq D_{R}^{2} H=2$, so $A^{2}=1$ and $\Delta(Y, A)=\operatorname{dim} Y+A^{2}-h^{0}(Y, A)=0$. Hence [Fuj, theorem 1.5.10] implies that $Y=\mathbf{P}^{2}$ and $A=\mathcal{O}_{\mathbf{P}^{2}}(1)$. Then $\mathcal{E}(1)$ is globally generated and it comes from the sequence:

$$
0 \longrightarrow \mathcal{O}_{\mathbf{P}^{2}}(-2) \longrightarrow \mathcal{O}_{\mathbf{P}^{2}}^{3} \longrightarrow \mathcal{E}(1) \longrightarrow 0 .
$$

Remark. It is convenient but not strictly necessary to use classification of contractions from smooth 3-folds. Instead it is sufficient to remark that if $A^{2}=2$ we have $\Delta(Y, A)=1$ and as above $A$ is globally generated. Then $\mathcal{E}$ is as above, which is impossible.

b) $\phi$ is divisorial. Let $E$ be the exceptional set of $\phi$; $E$ is a positive multiple of $\xi+v H, v \in \mathbf{Q}$.

1. $\operatorname{dim} \phi(E)=0$. Then $D_{R} E=0$, so $c_{2}=-u^{2}>0$, a contradiction.

2. $\operatorname{dim} \phi(E)=1$. Applying theorem 4.2 we obtain $u=1$ and from $D_{R}^{3}=3-c_{2}>0, c_{2} \leq 2$. This case is easy-see [SzW2, 2.3].

A.2. Classification of rank 2 Fano bundles on $\mathbf{P}^{3}$ follows from theorems 4.4, 5.2 and 6.2 in proofs of which we used only "easy" part of classification of these bundles-when $\mathcal{E}$ is normalized and $c_{2} \leq 1$. Note that in fact proofs can be simplified in case of bundles. Namely, divisorial and fiber contractions can be obtained only by computing Chern classes and small contractions can be derived only from equality (6.2.2.2), which reduces to the equality $4 d^{4}-d^{3}+1=0$ which does not have integral solutions. Also classification of Fano bundles on $\mathbf{Q}_{3}$ is much easier than that of 
bundles: we can easily compute all possible Chern classes (note that it was really the problem when only vector bundle techniques were used- $[\mathrm{SzW} 1])$. The classification of other ruled 4-folds is described in the paper (relevant computations are much easier than for sheaves).

\section{REFERENCES}

[AO] V. Ancona and G. Ottaviani, Some Applications of Beilinson's Theorem to Projective Spaces and Quadrics, Forum Math., 3 (1991), 157-176.

[A] T. Ando, On extremal rays of higher dimensional varieties, Invent. math., 81 (1985), 347-357.

[AW1] M. Andreatta and J. Wiśniewski, A note on non-vanishing and applications, Duke Math. J., 72 (1993), 739-755.

[AW2] M. Andreatta and J. Wiśniewski, On contractions of smooth varieties, MPI für Mathematik Bonn preprint, 96-62.

[Be] A. Beilinson, Coherent sheaves on $P^{n}$ and problems of linear algebra, Functional Anal. Appl., 12 (1978), 214-216, (English translation).

[BW] E. Ballico and J. Wiśniewski, On Bănică sheaves and Fano manifolds, Compositio Mathematica, 102 (1996), 313-335.

[ES] L. Ein and I. Sols, Stable vector bundles on quadric hypersurfaces, Nagoya Math. J., 96 (1984), 11-22.

[Fuj] T. Fujita, Classification theories of polarized varieties, London Lect. Notes, 115 (1990), Cambridge Press.

[Ful] W. Fulton, Intersection Theory, Ergebn. d. Math. u. ihr, Grenzg. 3 Folge-Band 2 (1984), Springer-Verlag.

[GH] Ph. Griffiths and J. Harris, Principles of algebraic geometry, Wiley, 1978.

[Ha1] R. Hartshorne, Algebraic Geometry, Springer-Verlag, 1977.

[Ha2] - Ample Vector Bundles, Publ. Math. IHES, 29 (1966).

[SRS1] Stable reflexive sheaves, Math. Ann., 254 (1980), 121-176.

[SRS2] L Stable reflexive sheaves II, Invent. math., 66 (1982), 165-190.

[SRS3] L Stable reflexive sheaves III, Math. Ann., 279 (1988), 517-534.

[Ka] Y. Kawamata, Small contractions of four dimensional algebraic manifolds, Math. Ann., 284 (1989), 595-600.

[KMM] Y. Kawamata, K. Matsuda and K. Matsuki, Introduction to the minimal model problem, Proc. Sendai Conf. Adv. in Pure Math., 10 (1987), 283-360.

[L] M. Lübke, Chernclassen von Hermite-Vektorbündlen, Math. Ann., 260 (1982), 133-141.

[OSS] Ch. Okonek, M. Schneider and H. Spindler, Vector bundles on complex projective spaces, Progress in Math., 3 (1980), Birkhäuser.

[PSW1] Th. Peternell, M. Szurek and J. Wiśniewski, Numerically effective vector bundles with small Chern classes, Lecture Notes in Math. 1507, Complex Algebraic Varieties, Bayreuth, 1990, Springer-Verlag (1992), 175-213.

[PSW2] — Fano manifolds and vector bundles, Math. Ann., 294 (1992), 151-165. 
[Re] M. Reid, Projective morphisms according to Kawamata, Warwick preprint (1983).

[SS] B. Shiffman and A. J. Sommese, Vanishing theorems on complex manifolds, Birkhäuser, 1985.

[SzW1] M. Szurek and J. Wiśniewski, Fano bundles over $\mathbf{P}^{3}$ and $\mathbf{Q}_{3}$, Pac. J. of Math., 141 (1990), 197-208.

[SzW2] — Fano bundles of rank 2 on surfaces, Compositio Math., 76 (1990), 295-305.

[SzW3] - On Fano manifolds which are $\mathbf{P}^{k}$-bundles over $\mathbf{P}^{2}$, Nagoya Math. J., 120 (1990), 89-101.

[SzW4] Conics, conic fibrations and stable bundles of rank 2 on some Fano threefolds, Rev. Roumaine Math. Pures Appl., 38 (1993), 729-741.

[W1] J. Wiśniewski, Ruled Fano 4-folds of index 2, Proc. of the AMS, 105 (1989), $55-61$.

[W2] $\longrightarrow$ A report on Fano manifolds of middle index and $b_{2} \geq 2$, preprint in Mathematica Gotingensis, Schriftenreihe des SFB, Geometrie u. Analysis, Heft, 16 (1993).

[W3] - Fano manifolds and quadric bundles, Math. Z., 214 (1993), 261-271.

Adrian Langer, Instytut Matematyki UW,

ul. Banacha 2, 02-097 Warszawa

alan@mimuw .edu.pl 